\title{
Constitutive activation of SHP2 in mice cooperates with ICSBP deficiency to accelerate progression to acute myeloid leukemia
} Iwona Konieczna,1,2 Elizabeth Horvath,1,2,3 Hao Wang, ${ }^{1,2}$ Stephan Lindsey, ${ }^{1,2}$ Gurveen Saberwal,1,2,3
Ling Bei,,1,3 Weiqi Huang, ${ }^{1,2}$ Leonidas Platanias,,$^{1,2,3}$ and Elizabeth A. Eklund, $1,2,3$

${ }^{1}$ Northwestern University Feinberg School of Medicine, Chicago, Illinois, USA. ${ }^{2}$ Robert H. Lurie Comprehensive Cancer Center of Northwestern University, Chicago, Illinois, USA. 3Jesse Brown Veteran's Health Administration Medical Center, Chicago, Illinois, USA.

\begin{abstract}
Myeloproliferative disorders (MPDs) are characterized by cytokine hypersensitivity and apoptosis resistance. Development of a block in myeloid differentiation is associated with progression of MPD to acute myeloid leukemia (AML) and portends poor prognosis. Identifying molecular markers of this transition may suggest targets for therapeutic intervention. Interferon consensus sequence binding protein (ICSBP, also known as IRF8) is an interferon-regulatory transcription factor that functions as a leukemia tumor suppressor. In mice, ICSBP deficiency induces an MPD that progresses to AML over time, suggesting that ICSBP deficiency is sufficient for myeloproliferation, but additional genetic lesions are necessary for AML. Since activity of ICSBP is influenced by tyrosine phosphorylation state, we hypothesized that mutations in molecular pathways that regulate this process might synergize with ICSBP deficiency for progression to AML. Consistent with this, we found that constitutive activation of SHP2 protein tyrosine phosphatase synergized with ICSBP haploinsufficiency to facilitate cytokine-induced myeloproliferation, apoptosis resistance, and rapid progression to AML in a murine bone marrow transplantation model. Constitutive SHP2 activation cooperated with ICSBP deficiency to increase the number of progenitors in the bone marrow and myeloid blasts in circulation, indicating a block in differentiation. Since SHP2 activation and ICSBP deficiency may coexist in human myeloid malignancies, our studies have identified a molecular mechanism potentially involved in disease progression in such diseases.
\end{abstract}

\section{Introduction}

The interferon consensus sequence binding protein (ICSBP, also referred to as IRF8) is a member of the interferon-regulatory factor (IRF) family of transcription factors (1). Previous studies determined that ICSBP is expressed exclusively in myeloid and B cells and regulates transcription of a number of genes involved in the inflammatory response. For example, in phagocytic cells, ICSBP activates transcription of genes encoding the NADPH oxidase proteins gp91 ${ }^{\text {PHOX }}$ and $\mathrm{p} 67^{\mathrm{PHOX}}$ (the CYBB and NCF2 genes, respectively), Toll-like receptor 4, and IL-12 (2-5). These genes are expressed late in myelopoiesis and confer phagocyte functional competence. ICSBP also activates transcription of NF1, the gene encoding the Ras-GAP neurofibro$\min 1$ (Nf1) (6-8). Nf1 deficiency results in cytokine hypersensitivity in murine models and human diseases. Therefore, decreased Nf1 expression is one mechanism for cytokine hypersensitivity in ICSBPdeficient cells $(6,7,9)$. Based on these results, ICSBP deficiency would be anticipated to impair differentiation and sustain proliferation during myelopoiesis. These activities would be consistent with the function of ICSBP as a myeloid leukemia suppressor.

Nonstandard abbreviations used: AML, acute myeloid leukemia; CML, chronic myeloid leukemia; ICSBP, interferon consensus sequence binding protein; IRF, interferon-regulatory factor; IVT, in vitro translation/translated; MPD, myeloproliferative disorder; NBT, nitroblue tetrazolium; PMN, neutrophil; PTP, protein tyrosine phosphatase; tMDS, therapy-related myelodysplastic syndrome.

Conflict of interest: The authors have declared that no conflict of interest exists. Citation for this article: J. Clin. Invest. 118:853-867 (2008). doi:10.1172/JCI33742.
ICSBP interacts with cis elements conforming to ISRE, PRDI, or composite ets/IRF consensus sequences (10). ICSBP has been described as being either an activator or repressor of gene transcription, depending on context. We previously found that ICSBP activates composite ets/IRF cis elements in the CYBB and NCF2 genes by interacting with a DNA-bound PU.1/IRF1 heterodimer. This interaction requires phosphorylation of conserved tyrosines in the IRF domains of ICSBP and IRF1 (3). Similarly, ICSBP activates an NF1 ets/IRF cis element by interacting with a DNA-bound PU.1/IRF2 heterodimer. This interaction requires phosphorylation of conserved IRF domain tyrosines in IRF2 and ICSBP (8). ICSBP also represses transcription of PRDI cis elements in undifferentiated myeloid cells (11). However, such cis elements are activated by interaction of tyrosine-phosphorylated ICSBP with DNA-bound IRF1 (11). ICSBP expression levels are relatively constant during myelopoiesis, but ICSBP tyrosine phosphorylation increases as differentiation proceeds $(2,7,9,11)$. Therefore, regulation of ICSBP tyrosine phosphorylation provides a mechanism for differentiation stage-specific target gene transcription. However, the role of tyrosine phosphorylation in mediating the leukemia-suppressing effects of ICSBP has not been investigated. This is addressed in the current studies.

In contrast, a number of studies suggest that the myeloid leukemia suppressor effect of ICSBP depends upon expression level. For example, ICSBP expression is decreased in $\mathrm{CD} 34^{+}$cells from subjects with therapy-related myelodysplastic syndrome/acute myeloid leukemia (tMDS/AML) in comparison to control subjects 
A

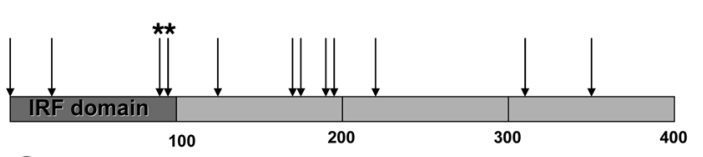

C

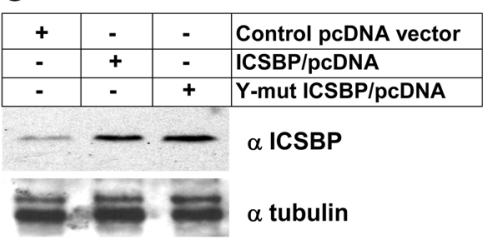

Western Blot U937 cell lysate proteins

D

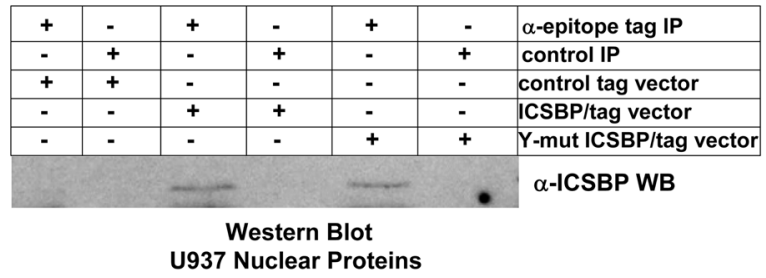

B

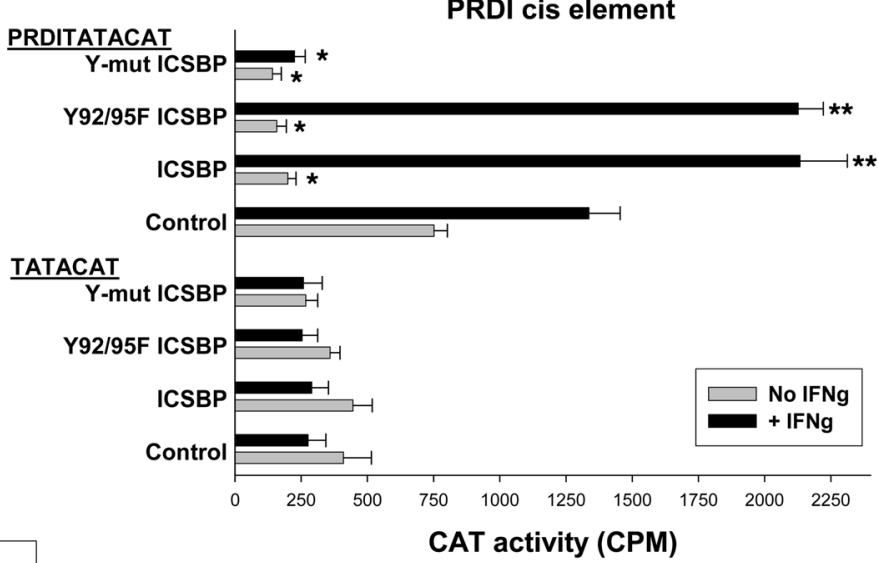

Figure 1

ICSBP tyrosine phosphorylation influenced target gene transcription. (A) Representation of tyrosine residues in ICSBP. The ICSBP amino acid sequence contains 12 tyrosine residues, including 2 highly conserved residues in the IRF domain (indicated by *). (B) The switch from PRDI cis element repression to activation during myelopoiesis is mediated by non-IRF domain ICSBP tyrosines. U937 cells were transfected with an artificial promoter construct containing 4 copies of the PRDI consensus (PRDITATACAT) or control (TATACAT) and a vector to express ICSBP; ICSBP with mutation of conserved IRF domain tyrosines (Y92/95F ICSBP); or all 12 tyrosines (Y-mut ICSBP). Reporter assays were performed with or without IFN $-\gamma$. The PRDI cis element was equivalently repressed by all forms of ICSBP in undifferentiated transfectants (statistically significant results are indicated by $\left.{ }^{*} P \leq 0.04\right)$. Differentiation increased PRDI cis element activity in transfectants with ICSBP, Y92/95F ICSBP, or vector control. This increase was not significantly different with WT versus Y92/95F ICSBP $\left({ }^{* *} P \leq 0.04\right)$. Repression activity of $Y$-mut ICSBP was not significantly altered by IFN- $\gamma$. (C) WT and Y-mut ICSBP were equivalently expressed in U937 transfectants. U937 cells were stably transfected with a vector to express ICSBP or Y-mut ICSBP, or vector control. Total lysate proteins were analyzed by Western blotting for ICSBP and tubulin (as a loading control). (D) WT and Y-mut ICSBP were similarly expressed in the nucleus. U937 cells were stably transfected with a vector to express epitope-tagged WT or Y-mut ICSBP, or vector control. Nuclear proteins from these transfectants were immunoprecipitated with an anti-ICSBP antibody and Western blotting performed with an anti-epitope tag antibody.

(12). Other studies found decreased ICSBP expression in bone marrow cells from subjects with uncontrolled or progressive chronic myeloid leukemia (CML), which normalized with remission (13, 14). To investigate the role of ICSBP in myelopoiesis, a murine model was developed with disruption of the IRF8 gene (15). Homozygous ICSBP-knockout mice develop a myeloproliferative disorder (MPD) with $100 \%$ penetrance. By 6 months, $80 \%$ of ICSBP-/- mice exhibit profound leukocytosis with myeloid blasts, which leads to death in $40 \%$ by 10 months. ICSBP-haploinsufficient mice develop mild MPD, but rarely myeloid blasts in the circulation (15).

These results suggested that ICSBP deficiency was sufficient for MPD, but additional genetic lesions were required for differentiation block and AML. Since posttranslational modification regulates ICSBP function, we hypothesized that lesions in pathways regulating this process might augment the impact of ICSBP deficiency on myelopoiesis. We previously found that ICSBP is a substrate for SHP1 or SHP2 protein tyrosine phosphatases (PTPs) in myeloid progenitors $(3,7)$. Although SHP1 or SHP2 protein abundance did not decrease during myelopoiesis, ICSBP was not a substrate for these PTPs in differentiating cells $(3,7)$. The mechanism for inactivation of these PTPs during myelopoiesis is unknown. However, mutations in the gene encoding SHP2 were recently described in human AML and MDS (16-18). Such mutations induce a conformation change that unmasks the PTP domain, resulting in constitutive SHP2 activation. We found that such SHP2 mutants dephosphorylate ICSBP throughout myelopoiesis and impair ICSBP target gene transcription $(7,19)$.

These results suggested that constitutive SHP2 activation might synergize with ICSBP deficiency and lead to disease progression and AML. A previous study identified ICSBP deficiency in tMDS/AML, and another study found activating mutations of SHP2 in this disease. The bcr/abl kinase activates SHP2, indicating that ICSBP deficiency and constitutive SHP2 activation may coexist in CML $(14,20,21)$. Potential coexistence of these 2 leukemia-associated abnormalities in human myeloid malignancies suggests they may be functionally significant for disease progression. This hypothesis was investigated in vitro and in vivo in the current studies.

\section{Results}

ICSBP tyrosine phosphorylation influenced gene transcription. The hypothesis of these studies was that phosphorylation of key ICSBP tyrosine residues is necessary for normal myelopoiesis. We planned to investigate this hypothesis by in vitro and in vivo studies using a tyrosine mutant form of ICSBP. We previously found that activation of 
A

\begin{tabular}{|c|c|c|c|c|c|c|c|c|c|c|c|c|}
\hline+ & + & + & + & - & - & - & - & - & - & - & - & $1 / 10$ input protein \\
\hline- & - & - & - & + & + & + & + & - & - & - & - & $\alpha-P Y$ IP \\
\hline - & - & - & - & - & - & - & - & + & + & + & + & Control IP \\
\hline+ & + & - & - & + & + & - & - & + & + & - & - & IVT ICSBP \\
\hline- & - & + & + & - & - & + & + & - & - & + & + & IVT Y-mut ICSBP \\
\hline- & + & - & + & - & + & - & + & - & + & - & + & Yop PTP \\
\hline - & - & $=$ & $=$ & - & 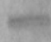 & & & & & & & Auto-radiograph \\
\hline
\end{tabular}

B

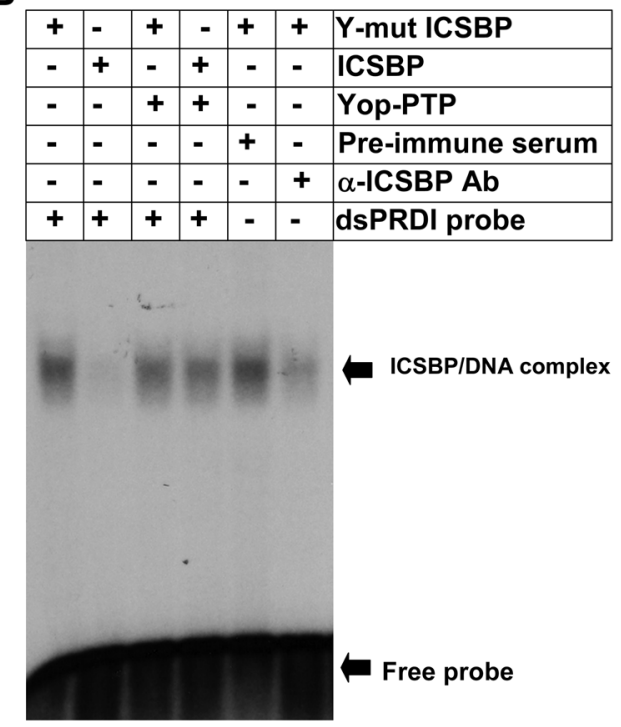

Figure 2

ICSBP bound the PRDI cis element in a tyrosine phosphorylation-dependent manner. (A) IVT WT ICSBP is tyrosine phosphorylated. IVT WT or Y-mut ICSBP was evaluated for tyrosine phosphorylation with or without treatment with recombinant Yop-PTP. Tyrosine phosphorylation of IVT ICSBP was decreased by this treatment, and Y-mut ICSBP was not tyrosine phosphorylated under either condition. (B) ICSBP binds the PRDI cis element in vitro in a tyrosine phosphorylation-dependent manner. IVT WT and Y-mut ICSBP were used in EMSA with a probe representing the PRDI consensus, with or without pretreatment with Yop-PTP. Y-mut ICSBP bound this probe with or without Yop treatment. In contrast, only Yop-treated WT ICSBP interacted with this probe in EMSA.

composite ets/IRF cis elements in some ICSBP target genes required phosphorylation of conserved tyrosine residues in the ICSBP IRF domain (Y92/95) (Figure 1A). These residues mediate interaction of ICSBP with PU.1 and IRF1 or IRF2 $(3,8)$. However, it was possible that additional tyrosine residues were involved in regulation of other target genes. For example, ICSBP tyrosine residues involved in the differentiation stage-specific functional switch from repression to activation of PRDI cis elements had not been identified (11).

To determine whether regulation of PRDI cis elements involved the conserved IRF domain tyrosines, we used an artificial promoter construct with multiple copies of the PRDI consensus sequence linked to a minimal promoter and reporter (referred to as PRDITATACAT). This construct (or TATACAT control) was cotransfected into U937 cells with a vector to express ICSBP, Y92/ 95F ICSBP (IRF domain tyrosine mutant), Y-mut ICSBP (ICSBP with all 12 tyrosine residues mutated to phenylalanine), or expression vector control. Reporter gene assays were performed with or without IFN- $\gamma$ differentiation. We used U937 myeloid leukemia cells for these studies because treatment of these cells with various cytokines, including IFN- $\gamma$, results in acquisition of characteristics of mature phagocytes (22). These characteristics include respiratory burst activity, proliferation arrest, and eventual apoptosis $(2,3)$. Of interest for our studies, ICSBP, IRF1, and IRF2 are tyrosine phosphorylated during U937 differentiation $(2,3,11)$.

In undifferentiated U937 transfectants, we found that overexpressed WT, Y92/95F, or Y-mut ICSBP repressed the PRDI cis element with equivalent efficiency $(P=0.5, n=4)$ (Figure $1 \mathrm{~B}$ ). Overexpressed WT or Y92/95F ICSBP equivalently activated the PRDI cis element in IFN- $\gamma$-differentiated transfectants $(P=0.9, n=4)$. In contrast, repression of the PRDI cis element by Y-mut ICSBP was not significantly different with or without differentiation
$(P=0.02, n=4)$. These results suggested that activation versus repression of the PRDI cis element was regulated by ICSBP tyrosines other than the conserved residues in the IRF domain.

In control experiments, we found that WT and Y-mut ICSBP protein were equivalently overexpressed in U937 transfectants (Figure 1C). We previously demonstrated that WT and Y92/95F ICSBP were equivalently overexpressed under these conditions (3, 7). In experiments with epitope-tagged proteins, we also found that overexpressed WT and Y-mut ICSBP were equivalently abundant in the nucleus of transfected cells (Figure 1D). This was consistent with our previous studies indicating that ICSBP is predominantly nuclear in U937 cells, with or without differentiation.

To identify the mechanism for sustained repression by Y-mut ICSBP in differentiating cells, we investigated the impact of tyrosine phosphorylation on binding to PRDI cis elements. Previous studies indicated that nonphosphorylated ICSBP bound PRDI cis elements directly, but tyrosine-phosphorylated ICSBP did not (11). We approached this question with DNA-binding assays using recombinant WT or Y-mut ICSBP. These proteins were generated by in vitro translation (IVT) in rabbit reticulocyte lysate. We previously found that IVT ICSBP was tyrosine phosphorylated and could be dephosphorylated by recombinant Yop-PTP (3). We performed initial experiments with ${ }^{35}$ S-labeled IVT WT or Y-mut ICSBP, with or without Yop-PTP treatment, to verify these results. As anticipated, WT ICSBP, but not Y-mut ICSBP, was immunoprecipitated by an anti-phospho-tyrosine antibody (Figure 2A). Consistent with our previous studies, incubation with Yop-PTP decreased abundance of tyrosine phosphorylated, but not total, WT ICSBP.

These proteins were used in EMSAs with a probe representing the PRDI consensus sequence (Figure 2B). We found that Y-mut ICSBP generated a shifted complex with this probe but WT ICSBP 
A

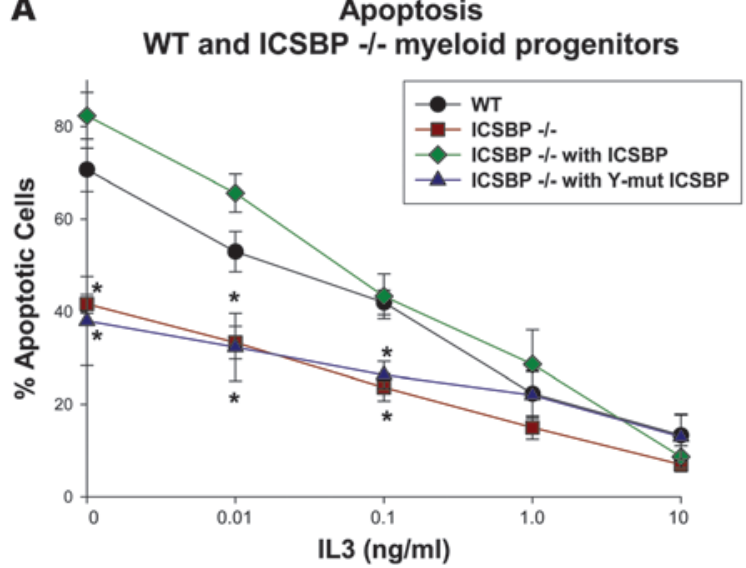

B

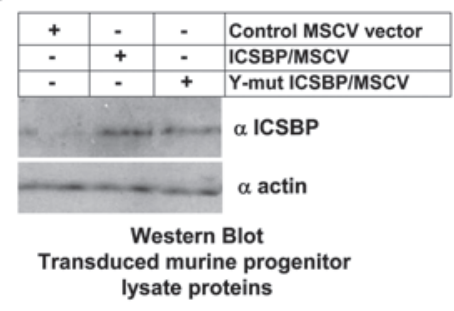

of control ICSBP I- $^{-/}$cells $(P>0.4$, $n=4)$. ICSBP and Y-mut ICSBP were equivalently expressed in $\mathrm{ICSBP}^{-/-}$murine myeloid progenitors (Figure $3 \mathrm{~B}$ ).

ICSBP tyrosine phosphorylation influenced development of $A M L$ in vivo. We were also interested in determining whether ICSBP tyrosine phosphorylation regulated myelopoiesis in vivo. We approached this question using a murine bone marrow transplantation model. For these studies, bone marrow was harvested from 8-week-old ICSBP-/mice, and $\mathrm{Sca} 1^{+}$cells were transduced with a vector to express WT or Y-mut ICSBP or control vector. This donor age was chosen because the bone marrow is in "proliferative phase" (pre-AML) (15). Transduced bone marrow was transplanted into at least 10 irradiated WT mice for each construct. Blood counts were determined every 4 weeks after transplantation for

did not. However, Yop-PTP-treated WT ICSBP bound to the PRDI probe. Since Y-mut ICSBP was not tyrosine phosphorylated and WT ICSBP was dephosphorylated by Yop-PTP, this was consistent with inhibition of PRDI binding by ICSBP tyrosine phosphorylation. The presence of ICSBP in the shifted complex was determined in control experiments with ICSBP antibody. Since ICSBP is tyrosine phosphorylated during myelopoiesis, these studies suggested that sustained direct PRDI binding of Y-mut ICSBP in differentiating myeloid cells resulted in sustained repression.

ICSBP tyrosine phosphorylation influenced apoptosis resistance. Although previous studies indicated that ICSBP-deficient cells were apoptosis resistant, the role of ICSBP tyrosine phosphorylation in apoptosis was not known. To investigate this, we studied myeloid progenitors during IL-3 withdrawal. For these studies, Sca1 $1^{+}$progenitors were isolated from the bone marrow of $\mathrm{ICSBP}^{-/-}$or WT control mice and granulocyte-monocyte progenitors (GMPs or GM$\mathrm{CFU}$ ) cultured in GM-CSF and IL-3 (7). ICSBP-/- progenitor cells were transduced with a retroviral vector to express ICSBP, Y-mut ICSBP, or vector control. As a positive control, WT progenitors were transduced with control vector. In previous studies, we found that either endogenous or overexpressed ICSBP was somewhat tyrosine phosphorylated under these cytokine conditions, although phosphorylation increased during ex vivo differentiation $(7,8)$.

Transduced cells were cultured for 48 hours in a dose titration of IL-3 and apoptosis determined by flow cytometry for annexin V staining. In comparison to WT cells, there were significantly fewer apoptotic ICSBP $-/$ cells at low IL-3 doses or in the absence of IL-3 (Figure $3 \mathrm{~A})(P<0.02, n=3)$. However, apoptosis in ICSBP ${ }^{-/}$cells that had been transduced with an ICSBP expression vector was not significantly different from that in WT cells $(P>0.1, n=3)$. In contrast, apoptosis of ICSBP-/- cells that were transduced with a vector to express Y-mut ICSBP was not significantly different from that
28 weeks. Cohorts of mice were sacrificed at 20 and 28 weeks for gene expression studies, as described in the next section.

We found that mice transplanted with control vector-transduced ICSBP $^{-/-}$bone marrow demonstrated an MPD that was consistent with previous observations in nontransplanted ICSBP-/- mice (15). These mice exhibited leukocytosis with a proportionately greater increase in neutrophils (PMNs) in comparison to lymphocytes (Figure 4A). Mature-appearing PMNs increased in these mice over 16 weeks and then decreased. This decrease coincided with appearance of myeloid leukemic blasts. In comparison, mice transplanted with ICSBP-/- bone marrow that had been transduced with a WT ICSBP expression vector exhibited significantly less leukocytosis $(P<0.0001, n=9)$ (Figure 4B). Although both PMNs and lymphocytes increased at later time points, there were significantly fewer circulating myeloid blasts throughout the observation period $(P<0.001, n=9)$. In contrast, leukocytosis and myeloid blasts in mice transplanted with Y-mut ICSBP expression vector-transduced ICSBP-/- bone marrow were not significantly different from those in mice transplanted with control ICSBP $^{-/-}$bone marrow $(P>0.2, n=9)$ (Figure 4C). Consistent with suppression of MPD, the spleens of mice transplanted with ICSBP expression vectortransduced ICSBP-/- bone marrow were significantly smaller than those of mice in the other 2 groups (Figure 4D).

Previous leukemogenesis studies in the ICSBP $-/$ murine model used morbidity or mortality as the end point (15). Such studies did not distinguish between death due to overwhelming MPD and death with progression to AML. In the current studies, we were interested in the impact of ICSBP tyrosine phosphorylation on transformation from MPD to differentiation block and AML. Therefore, instead of using death or moribundity as end points, we determined the percentage of circulating myeloid leukemic blasts as a quantitative measure of progression to AML. In initial studies, 
A

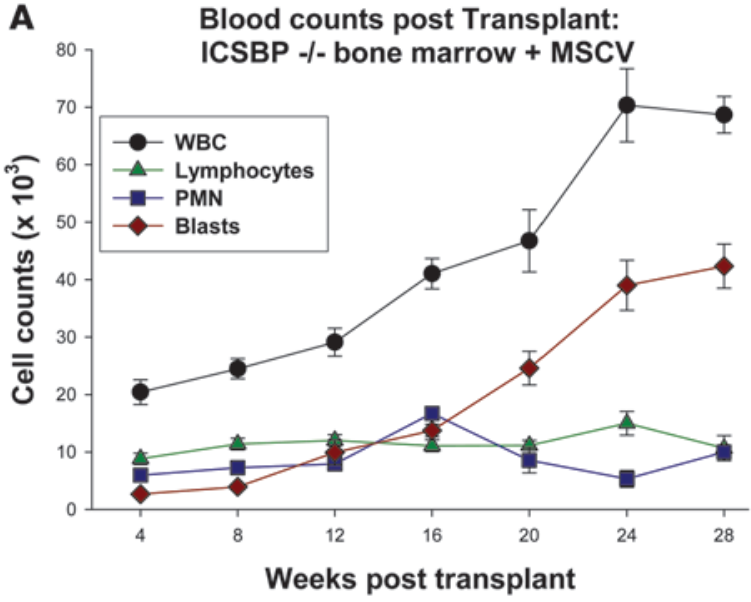

C

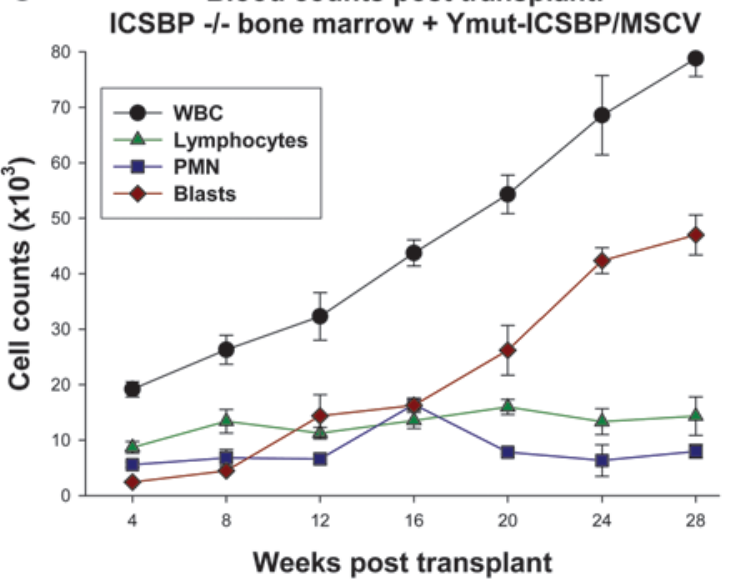

B

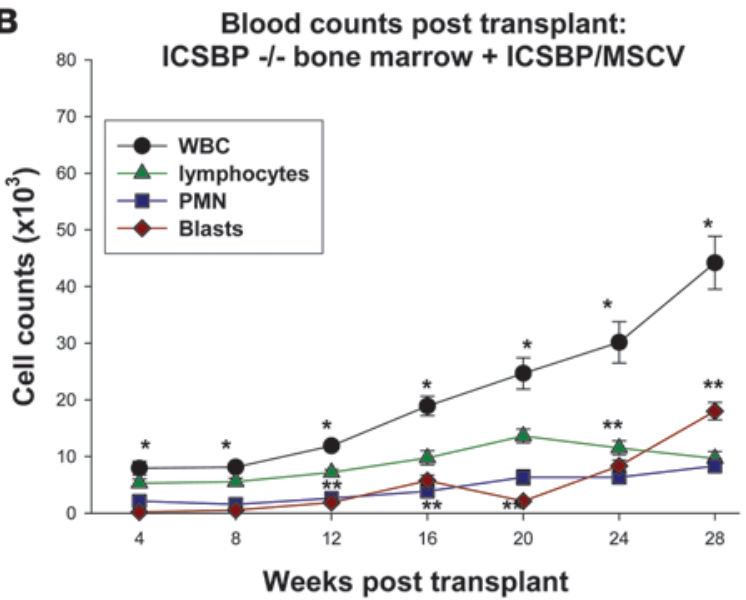

D

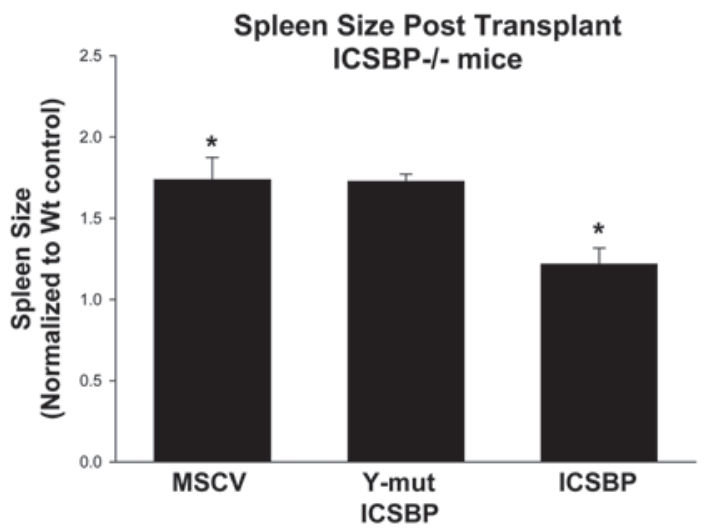

Figure 4

ICSBP tumor suppression required tyrosine phosphorylation. WT irradiated mice were transplanted with ICSBP-/- bone marrow that had been transduced with a retroviral vector to express ICSBP or Y-mut ICSBP, or control vector. Peripheral blood counts were determined every 4 weeks for 28 weeks. (A) MPD and AML developed in mice transplanted with control vector-transduced ICSBP-l- bone marrow. Control mice rapidly developed leukocytosis with neutrophilia. Numbers of myeloid blasts were equivalent to PMN numbers by 12 weeks and continued to increase throughout the observation period. (B) MPD and AML were delayed in mice transplanted with ICSBP ${ }^{-1-}$ bone marrow that had been transduced with an ICSBP expression vector. Total leukocytes were significantly reduced in these mice in comparison to mice transplanted with control or Y-mut ICSBP-expressing ICSBP ${ }^{-1-}$ bone marrow $\left({ }^{\star} P<0.0001\right)$. ICSBP reconstitution resulted in a normal, lymphocyte-dominant differential blood counts. Significantly fewer myeloid blasts were present in the circulation of these mice in comparison to the other 2 groups ( ${ }^{* *} P<0.001$ ). (C) MPD and AML developed in mice transplanted with ICSBP-l- bone marrow that had been transduced with a vector to express Y-mut ICSBP. These mice developed leukocytosis with neutrophilia and increasing myeloid blasts in a manner that was not significantly different than mice transplanted with control ICSBP $-1-$ bone marrow. (D) Splenomegaly was reduced in mice transplanted with ICSBP ${ }^{-/-}$bone marrow that was transduced with an ICSBP expression vector. Spleens harvested from mice 24 weeks after transplantation were significantly smaller in mice with ICSBP-reconstituted ICSBP-/- bone marrow in comparison to the other 2 groups.

we analyzed peripheral blood leukocyte counts in $\mathrm{WT}$, $\mathrm{ICSBP}^{+/}$, and ICSBP-/- mice to determine the onset of AML in this model in the absence of transplantation. A peripheral blast count of $20 \%$ was used to define AML. This peripheral blast count consistently correlated with approximately $30 \%$ blasts in the bone marrow. By this criteria, $50 \%$ of $\mathrm{ICSBP}^{-/-}$mice had AML by 16 weeks (Figure $5 \mathrm{~A}$ ). In contrast, very few myeloid blasts were seen in the circulation of $\mathrm{ICSBP}^{+/-}$mice throughout the observation period.

We also analyzed transplanted mice for progression from MPD to AML using the same criteria. We found that $50 \%$ of the mice transplanted with control or Y-mut ICSBP expression vector-transduced $\mathrm{ICSBP}^{-/}$- bone marrow exhibited at least $20 \%$ blasts in the peripheral circulation by 20 weeks (Figure 5B). In contrast, none of the mice transplanted with ICSBP $-/$ - bone marrow that had been transduced with WT ICSBP expression vector developed AML by 20 weeks, although some blasts were present at 28 weeks. Myeloid blasts were similar in appearance in the 3 groups of mice (Figure 5C).

These studies suggested that there was a difference in the impact of reconstituting ICSBP-/- bone marrow with WT versus Y-mut ICSBP. One possible explanation would be differences in expression of these 2 forms in vivo. To investigate this, bone marrow was harvested at 20 or 28 weeks, and ICSBP mRNA was quantitated by real-time PCR using primers that recognized the ICSBP transgene mRNA. At 20 weeks, we found equivalent expression of ICSBP transgene in mice transplanted with $\mathrm{ICSBP}^{-/-}$- bone marrow that had been transduced with either WT or Y-mut ICSBP 
A

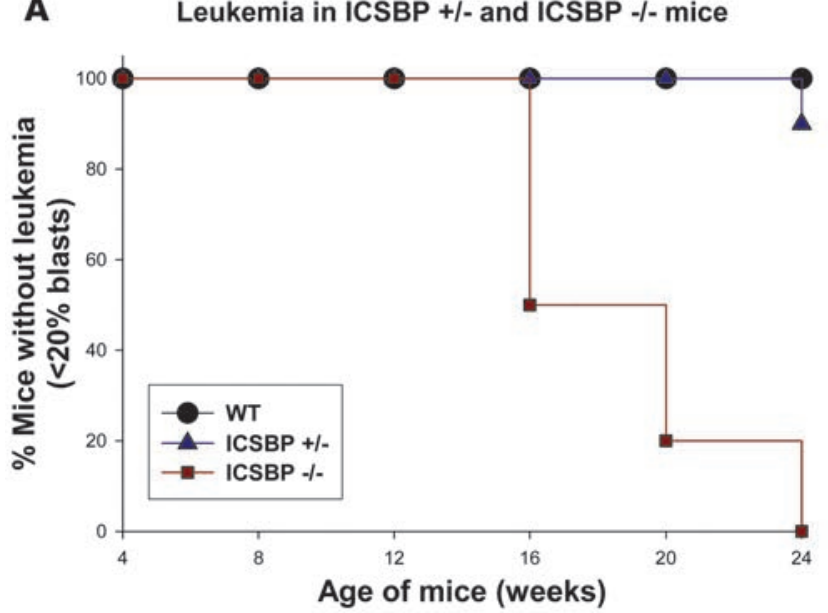

C Myeloid Blasts
B Leukemia post transplantation ICSBP -/- bone marrow

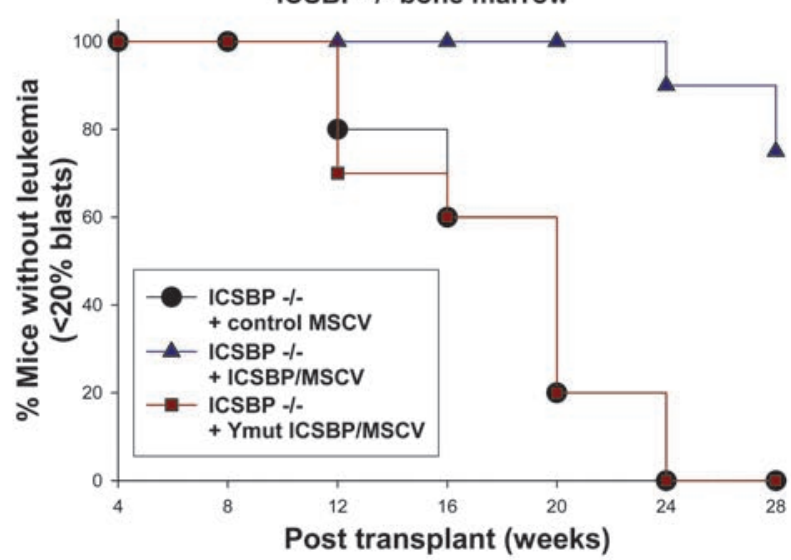

Post transplant (weeks)

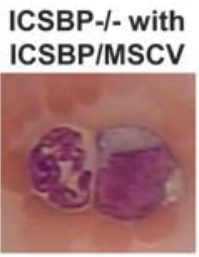

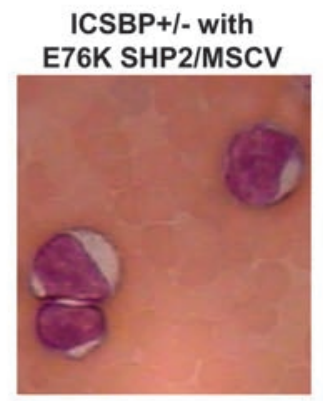

\section{Figure 5}

ICSBP protects from AML in a tyrosine phosphorylation-dependent manner. Development of leukemia in WT, ICSBP+/-, and ICSBP-/- mice or mice transplanted with ICSBP-I- bone marrow that was transduced with vectors to express WT or Y-mut ICSBP or vector control was monitored. Peripheral blood counts with leukocyte differential were determined every 4 weeks. Mice with greater than $20 \%$ myeloid blasts were considered to have AML. The percentage of mice without leukemia was plotted as a function of age. (A) AML developed by 6 months in ICSBP ${ }^{-1-}$ mice. Fifty percent of ICSBP ${ }^{-/-}$mice had AML by 16 weeks and $100 \%$ by 24 weeks. In contrast, only a few ICSBP ${ }^{+/-}$mice exhibited $20 \%$ blasts in the circulation by 24 weeks. (B) Expression of ICSBP, but not Y-mut ICSBP, delayed development of AML in mice transplanted with ICSBP-l- bone marrow. Fifty percent of mice transplanted with control vector or Y-mut ICSBP expression vector-transduced ICSBP ${ }^{-1-}$ bone marrow developed AML between 16 and 20 weeks. Two mice transplanted with ICSBP expression vector-transduced ICSBP-l- bone marrow developed AML during the observation period. (C) Myeloid blasts in transplanted mice. Peripheral smears of myeloid blasts from mice transplanted with ICSBP--- bone marrow that was transduced with vectors to express ICSBP or Y-mut ICSBP or control vector were compared with blasts from mice transplanted with ICSBP+/- bone marrow that had been transduced with a vector to express E76K SHP2. The blasts were morphologically similar. Original magnification, $\times 40$.

expression vector $(P=0.1, n=9)$ (Figure 6A). In mice transplanted with WT ICSBP expression vector-transduced ICSBP $-1-$ bone marrow, transgene expression decreased by 28 weeks $(P<0.01, n=9)$. This decrease correlated with an increase in total leukocytes and myeloid blasts. In contrast, ICSBP transgene expression increased over this time period in mice transplanted with Y-mut ICSBP expression vector-transduced ICSBP-/- bone marrow.

ICSBP tyrosine phosphorylation influenced hematopoietic stem cell expansion and differentiation in vivo. To provide additional characterization of the leukemia that developed in these animals, we determined the impact of WT versus Y-mut ICSBP on in vivo expansion of the HSC population and on differentiation. To investigate this, we analyzed bone marrow harvested from mice at 20 and 28 weeks after transplantation by real-time PCR. CD34 expression was determined as an indication of the relative size of the bone marrow HSC and immature progenitor pool (Figure 6B). We found that CD34 expression increased significantly between 20 and 28 weeks in mice transplanted with control or Y-mut ICSBP expression vector-transduced ICSBP $^{-} /$- bone marrow $(P<0.02$, $n=9)$ and was not significantly different in the 2 groups $(P>0.6$, $n=9)$. This expansion of the immature progenitor pool correlated with an increase in myeloid blasts in the circulation. CD34 expression was significantly lower at 20 weeks after transplantation with WT ICSBP expression vector-transduced ICSBP $-/$ - bone marrow $(P=0.05, n=9)$. CD34 expression increased in these latter mice between 20 and 28 weeks, correlating with decreased ICSBP transgene and increased myeloid blasts.

We investigated gp91 9 PHOX expression as a marker of progressive differentiation block. gp $91^{\text {PHOX }}$ is expressed exclusively after the promyelocyte stage of myelopoiesis and is also an ICSBP target gene. At 20 and 28 weeks, significantly more gp91 ${ }^{\text {PHOX }}$ was expressed in the bone marrow of mice transplanted with ICSBP expression vector-transduced $\mathrm{ICSBP}^{-/}$- bone marrow in comparison to the other 2 groups $(P=0.002, n=9)$ (Figure 6C). This correlated with increased circulating blasts and HSC expansion in mice transplanted with control or Y-mut ICSBP expression vector-transduced $\mathrm{ICSBP}^{-/-}$bone marrow. gp91 ${ }^{\mathrm{PHOX}}$ expression decreased between 20 and 28 weeks in mice transplanted with 
A

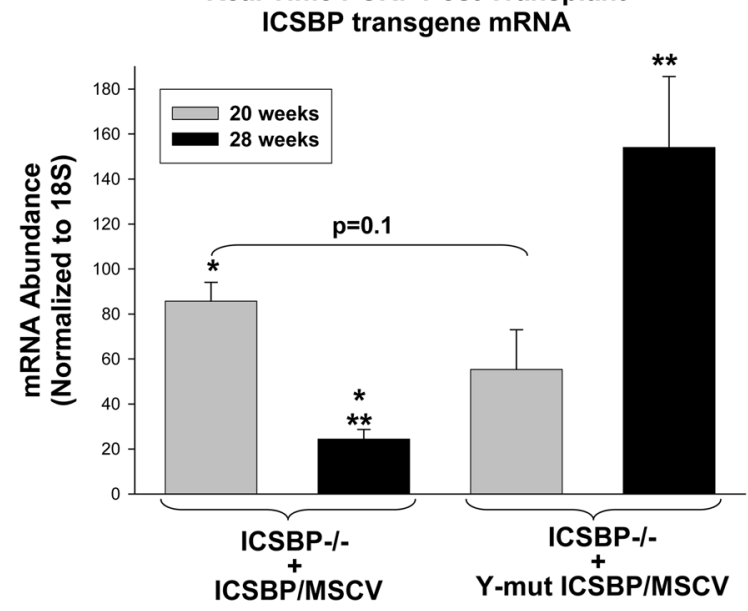

C

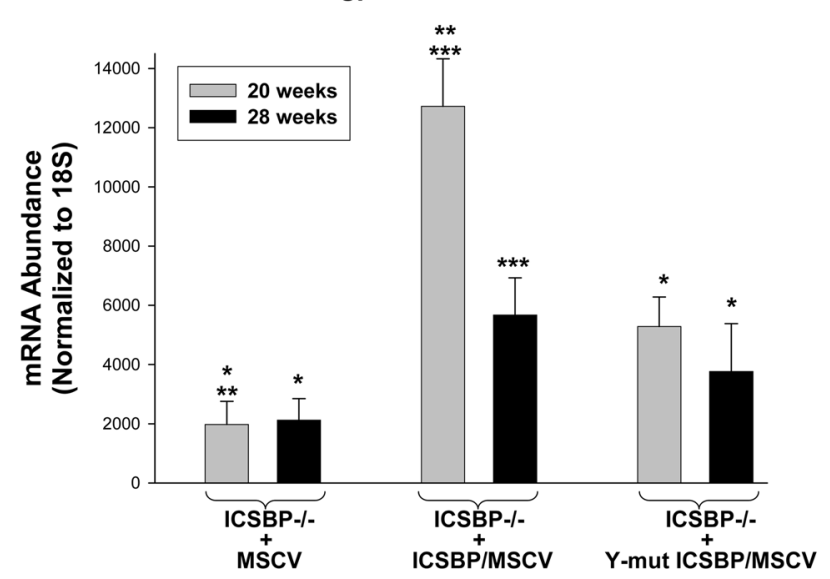

B

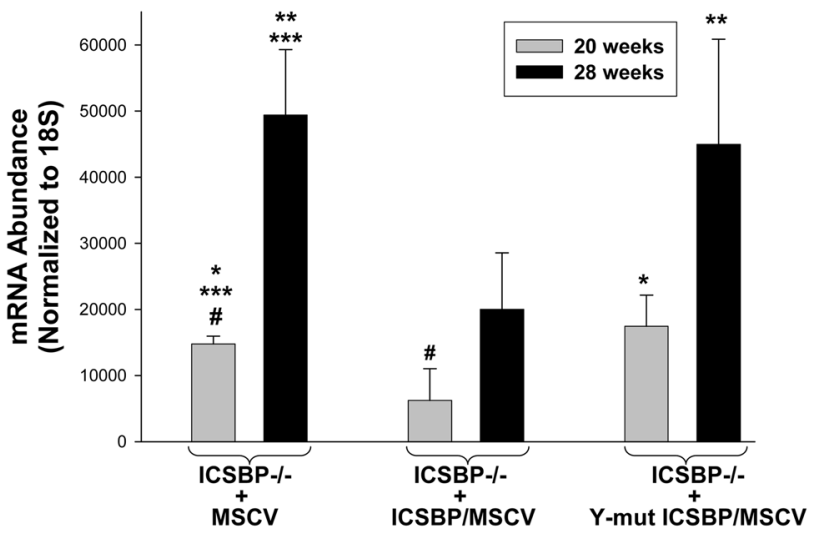

D

Figure 6

ICSBP tyrosine phosphorylation regulated differentiation and HSC expansion. (A) Expression of the ICSBP transgene. ICSBP transgene expression in mice transplanted with WT or Y-mut ICSBP-expressing ICSBP-l- bone marrow was determined by real-time PCR. Expression of these 2 transgenes was not significantly different at 20 weeks. WT transgene decreased significantly at 28 weeks $\left({ }^{\star} P<0.01\right)$. Wnt transgene expression was significantly less than $Y$-mut transgene expression $\left({ }^{\star \star} P<0.01\right)$. (B) Bone marrow CD34 expression. Bone marrow was analyzed by real-time PCR for CD34 expression. CD34 expression was not significantly different in control versus Y-mut ICSBP-expressing ICSBP $-1-$ bone marrow at $20\left({ }^{\star} P<0.001\right)$ or $28\left({ }^{* *} P<0.02\right)$ weeks but increased significantly over time $\left.{ }^{\star * \star} P<0.05\right)$. CD34 expression was significantly lower in mice transplanted with ICSBP-transduced ICSBP ${ }^{-l-}$ bone marrow $\left({ }^{\#} P<0.02\right)$. (C) Bone marrow gp91 ${ }^{\text {PHOX }}$ expression. Bone marrow gp91 ${ }^{\text {PHOX }}$ expression was determined by real-time PCR. Expression was not significantly different in mice transplanted with control or Y-mut ICSBP-expressing ICSBP $^{-/-}$bone marrow $\left({ }^{\star} P=0.002\right)$. Expression was significantly greater at 20 weeks in mice transplanted with ICSBP-expressing ICSBP $-/-$ bone marrow ( ${ }^{* \star} P=0.02$ ) but decreased significantly by 28 weeks $\left({ }^{* \star} P<0.01\right)$. (D) ICSBP deficiency impaired NADPH oxidase activity. Peripheral blood was obtained 16 weeks after transplantation and analyzed by NBT slide test. Results were expressed as percentage of NBT-positive PMNs (with or without PMA for oxidase activation). There were significantly more NBT-positive PMNs in mice transplanted with ICSBP-1- bone marrow reexpressing ICSBP than with control or Y-mut ICSBP-expressing bone marrow $\left({ }^{\star} P<0.01\right)$. E76K SHP2 expression in WT $\left({ }^{\star \star} P<0.01\right)$ or ICSBP $\left.+/{ }^{\star \star \star} P<0.01\right)$ bone marrow significantly decreased the percentage of NBT-positive PMNs.

ICSBP-expressing ICSBP-/- bone marrow $(P=0.02, n=9)$. This correlated with decreased WT ICSBP transgene, HSC expansion, and the emergence of blasts in the circulation.

gp91 ${ }^{\text {PHOX }}$ is a rate-limiting NADPH oxidase protein. To correlate gene expression with phenotype, we investigated peripheral blood PMNs for respiratory burst activity by nitroblue tetrazolium (NBT) slide test. The percentage of NBT-positive, morphologically mature PMNs was determined. We found relatively few NBTpositive PMNs in mice transplanted with control or Y-mut ICSBP expression vector-transduced $\mathrm{ICSBP}^{-/-}$bone marrow $(P=0.5$, $n=3$ ) (Figure 6D). There was a significantly greater percentage of NBT-positive PMNs in mice transplanted with $\mathrm{ICSBP}^{-/-}$bone marrow that had been transduced with a vector to reconstitute ICSBP expression $(P<0.01, n=3)$. These results suggested ICSBP deficiency was associated with impaired terminal differentiation.

Expression of constitutively active SHP2 influenced cytokine bypersensitivity and apoptosis in ICSBP-deficient cells. These studies suggested that impaired ICSBP tyrosine phosphorylation might cooperate with ICSBP deficiency to dysregulate myelopoiesis and lead to AML. We previously found that constitutively active SHP2 mutants impaired 
A

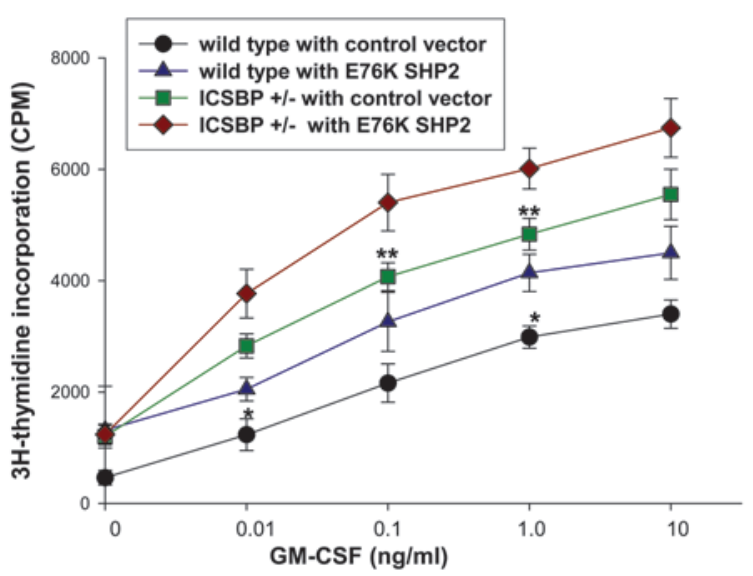

B Apoptosis

WT and ICSBP +/- myeloid progenitors

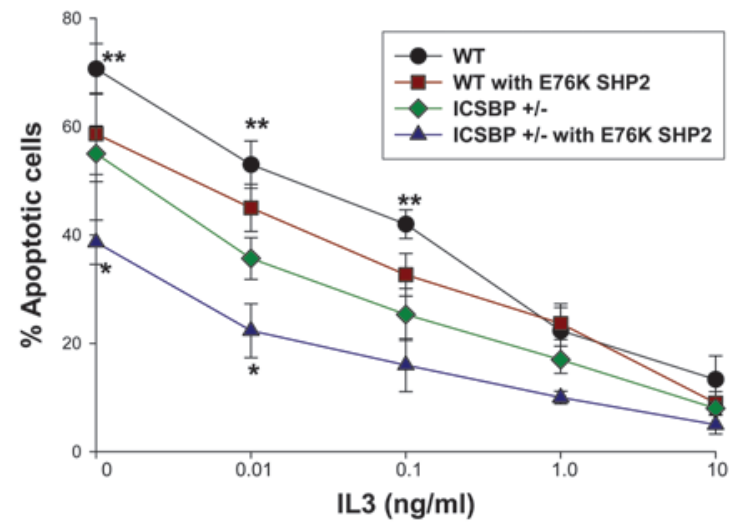

C

\begin{tabular}{|c|c|c|c|c|}
\hline+ & + & - & - & ICSBP +/- \\
\hline - & - & + & + & WT \\
\hline+ & - & + & - & Control MSCV vector \\
\hline- & + & - & + & E76K SHP2/MSCV \\
\hline \multicolumn{2}{|c|}{ n. } & 60 & 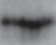 & $\alpha$-ICSBP \\
\hline \multicolumn{4}{|c|}{ 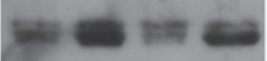 } & $\alpha-S H P 2$ \\
\hline & & & & $\alpha$-GAPDH \\
\hline
\end{tabular}

Western Blot

Transduced murine progenitor lysate proteins
D

\begin{tabular}{|c|c|c|c|c|c|c|c|c|}
\hline+ & + & + & + & - & - & - & - & $\alpha-P Y$ IP \\
\hline- & - & - & - & + & + & + & + & Control IP \\
\hline+ & + & - & - & + & + & - & - & ICSBP +/- \\
\hline- & - & + & + & - & - & + & + & Wt \\
\hline+ & - & + & - & + & - & + & - & Control MSCV vector \\
\hline- & + & - & + & - & + & - & + & E76K SHP2/MSCV \\
\hline - & - & - & - & & & & & $\alpha-I C S B P$ WB \\
\hline
\end{tabular}

\section{Figure 7}

Constitutive SHP2 activation increased cytokine hypersensitivity and apoptosis resistance in ICSBP-haploinsufficient myeloid cells. Myeloid progenitors were isolated from WT or ICSBP +- mice, cultured in GM-CSF and IL-3, and transduced with a vector to express E76K SHP2 or vector control. (A) Constitutively active SHP2 (E76K) increased GM-CSF hypersensitivity of myeloid progenitors. Proliferation assays of transduced cells were performed with a dose titration of GM-CSF. Proliferation was significantly increased at low GM-CSF doses in E76K SHP2-expressing WT or ICSBP ${ }^{+/-}$progenitor cells in comparison to control WT $\left({ }^{*} P<0.02\right)$ or ICSBP $+/-$ progenitors $\left({ }^{* *} P<0.02\right)$. (B) E76K SHP2 increased apoptosis resistance of ICSBP+/- myeloid progenitors. Transduced cells were cultured in a dose titration of IL-3 and apoptosis determined by annexin $\mathrm{V}$ staining. E76K SHP2 expression significantly decreased the percentage of apoptotic cells at low IL-3 doses in ICSBP $+/-$ cells ( $\left.{ }^{*} P<0.01\right)$ but not WT cells. WT progenitors exhibited significantly more apoptosis at low IL-3 doses than ICSBP+/- progenitors $\left({ }^{\star *} P<0.001\right)$. (C) E76K SHP2 was equivalently overexpressed in WT and ICSBP+/- cells. Transduced cells were analyzed for protein expression by Western blots serially probed with antibodies against SHP2, ICSBP, and GAPDH (as a loading control). SHP2 overexpression did not alter ICSBP expression. (D) Tyrosinephosphorylated ICSBP was less abundant in E76K SHP2-expressing ICSBP + -/ cells than in E76K SHP2-expressing WT cells. These transduced cells were also analyzed by anti-phospho-tyrosine immunoprecipitation followed by Western blots probed for ICSBP.

ICSBP tyrosine phosphorylation and target gene transcription during myeloid differentiation in vitro $(6,7,19)$. Both $\operatorname{ICSBP}^{-/-}$myeloid progenitors and WT progenitors expressing leukemia-associated, constitutively active SHP2-mutants are characterized by GM-CSF hypersensitivity $(6,7)$. We found that expression of such SHP2 mutants (E76K SHP2) did not increase GM-CSF hypersensitivity of $\mathrm{ICSBP}^{-/-}$progenitor cells. However, E76K SHP2 prevented reexpression of ICSBP from rescuing this aspect of the phenotype (7).

ICSBP is deficient, but not absent, in human myeloid malignancies. Therefore, we determined the impact of activated SHP2 mutants on GM-CSF hypersensitivity in ICSBP haploinsufficient cells. For these studies, myeloid progenitors were cultured from WT or ICSBP ${ }^{+/-}$bone marrow and transduced with a retroviral vector to express constitutively active SHP2 (E76K) or vector control. Cells were deprived of cytokines for 24 hours, and proliferation was determined in response to a GM-CSF dose titration. We found that expression of E76K SHP2 in WT progenitors significantly increased proliferation at low GM-CSF doses, consistent with previous results (Figure 7A) (7). $\mathrm{ICSBP}^{+/-}$progenitors exhibited significantly more proliferation than WT at low GM-CSF doses, and E76K SHP2 expression significantly increased proliferation of these progenitors at such doses $(P<0.02, n=4)$.

We also investigated the impact of constitutive SHP2 activation on apoptosis resistance in myeloid progenitors. For these studies, transduced WT or $\mathrm{ICSBP}^{+/-}$cells were treated with a dose titration of IL-3 for 48 hours, and apoptosis was determined by flow cytometry. We found that survival of $\mathrm{ICSBP}^{+/-}$progenitors was significantly greater than that of WT at low IL-3 doses $(P<0.01, n=4)$ (Figure 7B). We also found that E76K SHP2 significantly increased the number of $\mathrm{ICSBP}^{+/}$cells that survived at the lowest IL-3 doses $(P<0.01, n=4)$. Interestingly, E76K SHP2 did not significantly alter the survival of WT progenitor cells at any IL-3 dose. 
A Blood counts post transplant: ICSBP +/- bone marrow + MSCV

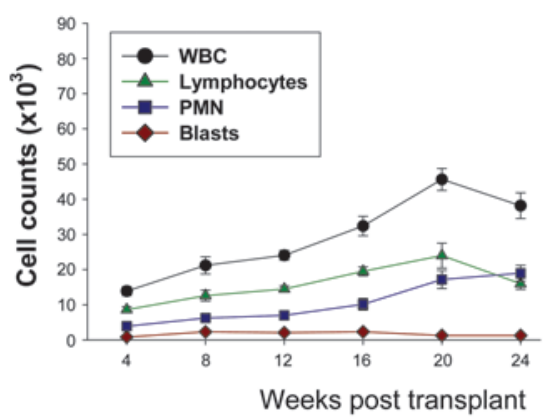
D Blood counts post transplant:
WT bone marrow + MSCV

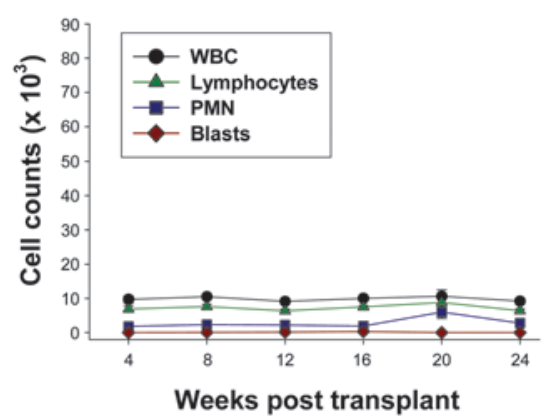

G Spleen Size Post Transplant

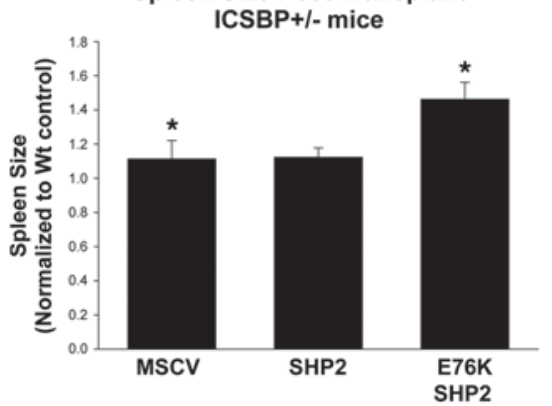

B Blood counts post transplant: ICSBP +/- bone marrow + SHP2/MSCV

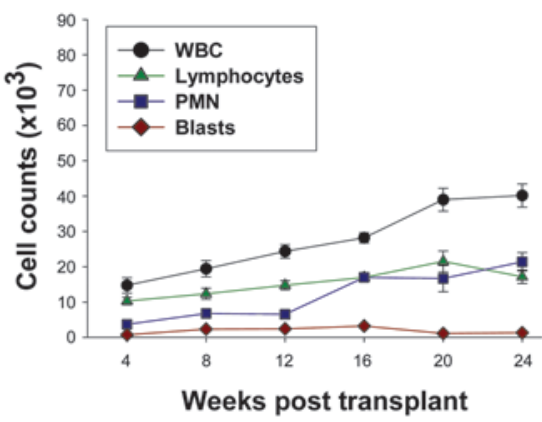

E

Blood counts post transplant: WT bone marrow + SHP2/MSCV

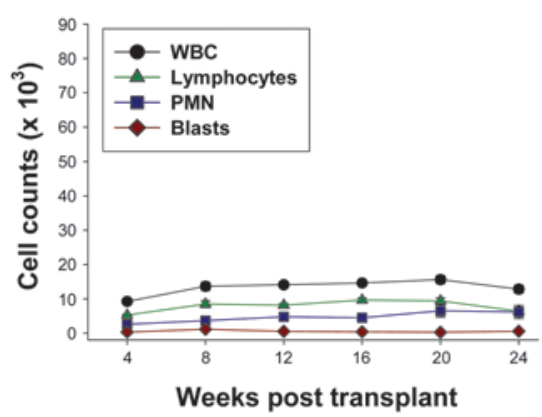

C Blood counts post transplant: ICSBP +/- bone marrow + E76K SHP2/MSCV

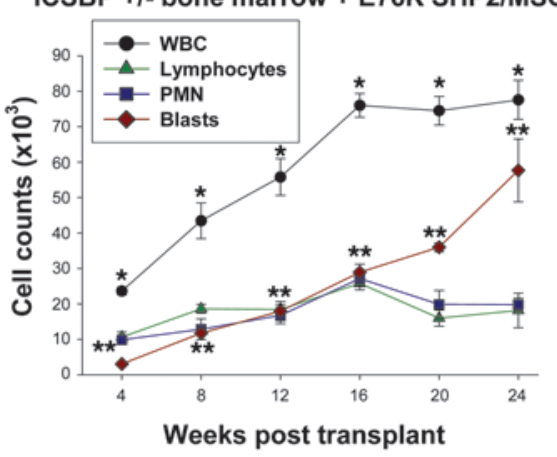

F Blood counts post transplant: WT bone marrow + E76K SHP2/MSCV

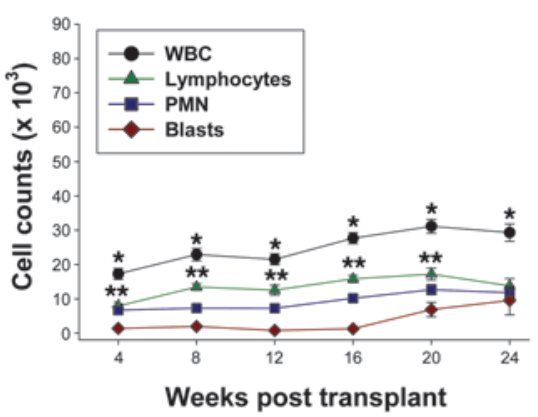

\section{Figure 8}

E76K SHP2 cooperated with ICSBP haploinsufficiency to accelerate AML. WT irradiated mice were transplanted with WT or ICSBP+/- bone marrow that was transduced with a retroviral vector to express SHP2, E76K SHP2, or control. Peripheral blood counts were determined every 4 weeks for 24 weeks. (A) Mild MPD developed in mice transplanted with control ICSBP+/- bone marrow. These mice developed mild neutrophilia, but rare myeloid blasts. (B) Mild MPD developed in mice transplanted with WT SHP2-overexpressing ICSBP $+/$ - bone marrow. These mice developed leukocytosis that was not significantly different from that in mice transplanted with control ICSBP+/- bone marrow. (C) MPD and rapid AML developed in mice transplanted with E76K SHP2-expressing-ICSBP + - bone marrow. These mice developed leukocytosis that was significantly greater than that in mice transplanted with control or WT SHP2-overexpressing ICSBP ${ }^{+/-}$bone marrow $\left({ }^{*} P<0.001\right)$ with significantly more myeloid blasts $\left({ }^{\star \star} P<0.01\right)$. (D) Peripheral blood counts were normal in mice transplanted with control vector-transduced WT bone marrow. These mice exhibited normal peripheral blood counts and leukocyte differential. (E) Peripheral blood counts were normal in mice transplanted with SHP2 expression vector-transduced WT bone marrow. These mice also exhibited normal peripheral blood counts and leukocyte differential. (F) Mild MPD developed in mice transplanted with E76K SHP2-expressing WT bone marrow. These mice exhibited significantly greater total leukocyte counts $\left({ }^{\star} P<0.001\right)$ than mice transplanted with control WT bone marrow with significant neutrophilia $\left({ }^{* *} P<0.001\right)$. (G) Mice transplanted with E76K SHP2-expressing ICSBP+/- bone marrow developed splenomegaly. Spleens harvested from these mice were significantly larger than those from mice transplanted with control or WT SHP2-overexpressing ICSBP + - bone marrow.

These results suggested that constitutive SHP2 activation influenced proliferation and apoptosis via altered ICSBP tyrosine phosphorylation. Alternatively, it was also possible that E76K SHP2 decreased ICSBP expression in myeloid progenitors. To investigate this, Western blots of lysate proteins from transduced cells were probed with antibodies against ICSBP, SHP2, and GAPDH (as a loading control). We found that E76K SHP2 expression did not alter ICSBP expression (Figure 7C). Our results also suggested that expression of E76K SHP2 had a differential impact on the abundance of tyrosine-phosphorylated ICSBP in WT versus $\mathrm{ICSBP}^{+/-}$cells. To verify this, the ICSBP tyrosine phosphorylation state in the transduced cells was determined. Lysate proteins were 
A

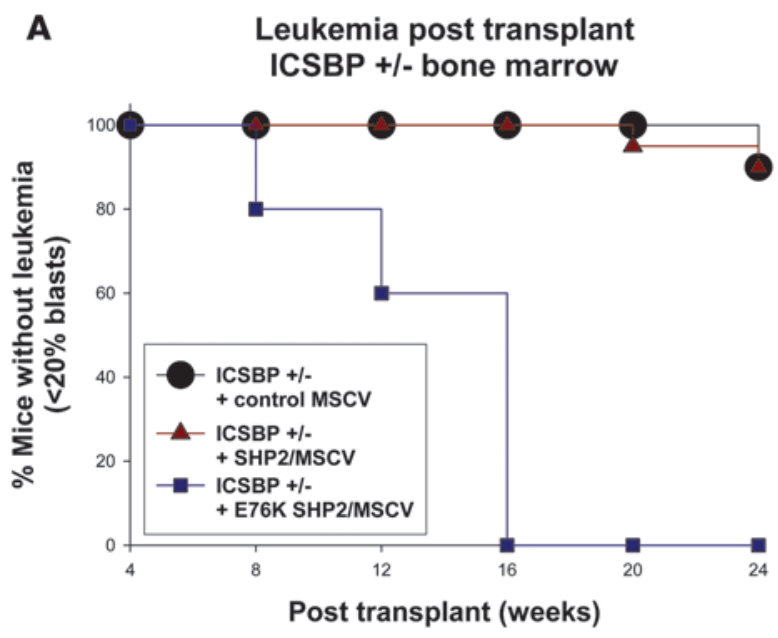

B

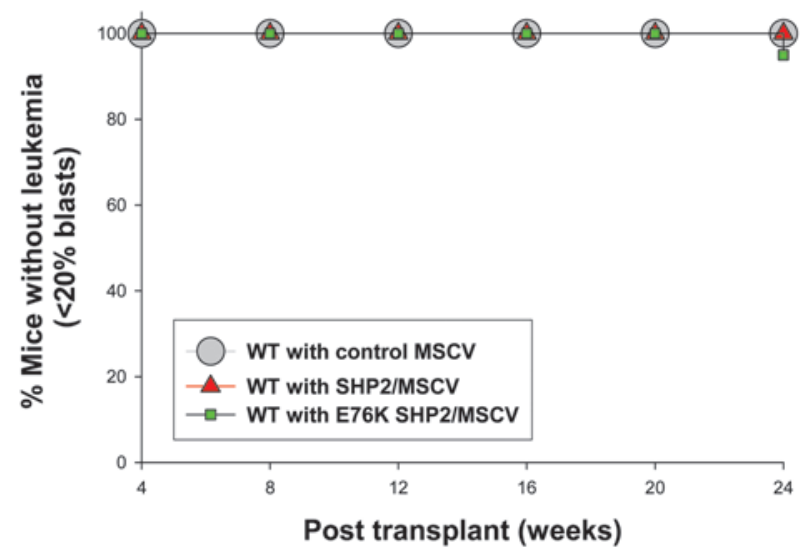

Figure 9

E76K SHP2 cooperated with ICSBP haploinsufficiency for AML. Development of leukemia in mice transplanted with WT or ICSBP+/- bone marrow that was transduced with vectors to express WT or E76K SHP2 or vector control was monitored as described above. Mice with greater than $20 \%$ myeloid blasts were considered to have AML. The percent of mice without leukemia was plotted as a function of age. (A) Mice transplanted with ICSBP +- - bone marrow that was transduced with a vector to express E76K SHP2 rapidly developed AML. Fifty percent of these mice developed AML between 12 and 16 weeks after transplantation. In contrast, only 2 mice transplanted with either control or WT SHP2-overexpressing $\mathrm{ICSBP}^{+/-}$bone marrow developed AML during the observation period. (B) AML did not develop in mice transplanted with WT bone marrow that was transduced with E76K SHP2 expression vector. Few of these mice developed AML by 24 weeks. None of the mice transplanted with WT bone marrow transduced with control or SHP2 expression vector developed AML during the observation period.

immunoprecipitated with a phospho-tyrosine antibody (under denaturing conditions), and Western blots of immunoprecipitates were probed with an ICSBP antibody (Figure 7D). We found that both total and tyrosine-phosphorylated ICSBP were decreased in E76K SHP2-expressing ICSBP ${ }^{+/-}$cells in comparison to WT cells expressing constitutively active SHP2.

Expression of constitutively active SHP2 cooperated with ICSBP deficiency to induce $A M L$ in vivo. Our studies suggested that constitutive SHP2 activation cooperated with ICSBP deficiency to dysregulate myeloid differentiation, cytokine-induced proliferation, and cell survival in vitro. Therefore, we tested cooperation between these 2 leukemia-associated abnormalities in vivo. For these studies, irradiated WT mice were transplanted with $\mathrm{WT}_{\text {or }} \mathrm{ICSBP}^{+/-}$bone marrow that had been transduced with a retroviral vector to express E76K SHP2, WT SHP2 (as a control for overexpression), or empty control vector. At least 10 mice were transplanted for each construct, and blood counts were determined every 4 weeks after transplantation. Mice were sacrificed at 16 and 24 weeks for gene expression studies.

We found that mice transplanted with control $\mathrm{ICSBP}^{+/-}$bone marrow developed a mild MPD with neutrophilia (Figure 8A), similar to nontransplanted $\mathrm{ICSBP}^{+/-}$mice. Few myeloid blasts were detected in the peripheral blood of these mice throughout the observation period. Leukocytosis and neutrophilia in mice transplanted with $\mathrm{ICSBP}^{+/-}$bone marrow that had been transduced with WT SHP2 expression vector were not significantly different from these control mice $(P>0.5, n=9)$ (Figure $8 \mathrm{~B})$. In contrast, mice transplanted with E76K SHP2 expression vector-transduced $\mathrm{ICSBP}^{+/-}$bone marrow developed profound leukocytosis with dominant PMNs $(P<0.0001, n=9)$ (Figure 8C). Significantly greater numbers of myeloid blasts were present in the peripheral blood of these mice at all time points in comparison to either control group $(P<0.0001, n=9)$.
Mice transplanted with WT bone marrow that had been transduced with control (Figure 8D) or SHP2 expression vector (Figure 8E) exhibited normal murine blood counts. A mild MPD developed in mice transplanted with E76K SHP2 expression vector-transduced WT bone marrow (Figure 8F). Total leukocytes, PMNs, and lymphocytes were present in significantly greater numbers in these mice in comparison to either WT control group $(P<0.001, n=9)$. Therefore, these studies indicated that constitutive SHP2 activation and ICSBP haploinsufficiency induced greater myeloproliferation in comparison to either alone. Consistent with this, spleens of mice transplanted with E76K SHP2 expression vector-transduced $\mathrm{ICSBP}^{+/}$- bone marrow were significantly larger than the spleens in the other groups of mice at 20 weeks (Figure 8G).

Other investigators found that mice that had been transplanted with WT bone marrow expressing activated SHP2 mutants developed a fatal MPD (18). This occurred with a time course similar to that in $\mathrm{ICSBP}^{-/-}$mice (15). In our studies, we were interested in the impact of constitutive SHP2 activation on differentiation block and AML in ICSBP-deficient hematopoiesis. Therefore, as discussed above, we did not use death or moribundity as end points, because these events may be due to overwhelming MPD or the consequences of AML. Instead, we specifically investigated this transition by quantifying peripheral blood myeloid blasts. We found that AML (>20\% peripheral blasts) developed by 16 weeks in $100 \%$ of mice transplanted with E76K SHP2 expression vectortransduced $\mathrm{ICSBP}^{+/-}$bone marrow (Figure 9A). We found a correlation between peripheral and bone marrow blasts similar to that observed in the studies described above. In contrast, AML did not develop in mice transplanted with control or WT SHP2 expression vector-transduced $\mathrm{ICSBP}^{+/}$bone marrow (Figure 9A) or in any of the mice transplanted with transduced WT bone marrow (Figure 9B). Therefore, expression of constitutively active SHP2 in ICSBP- 
A

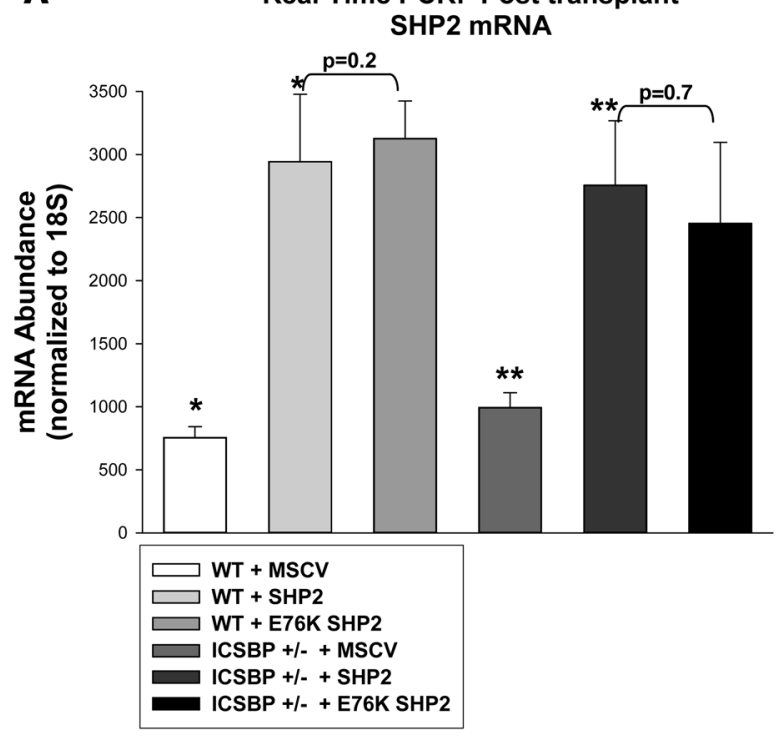

B

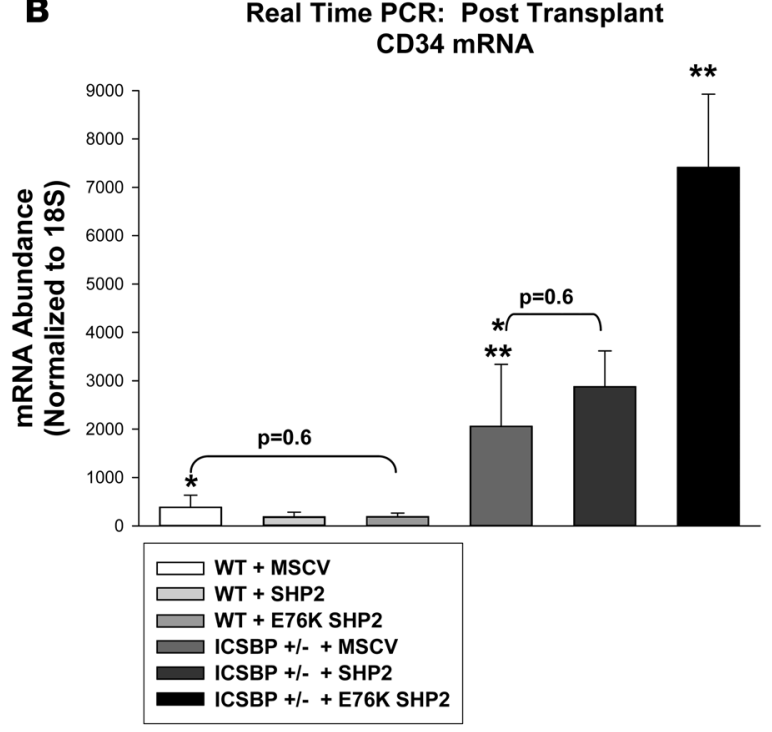

C

Real Time PCR: Post transplant
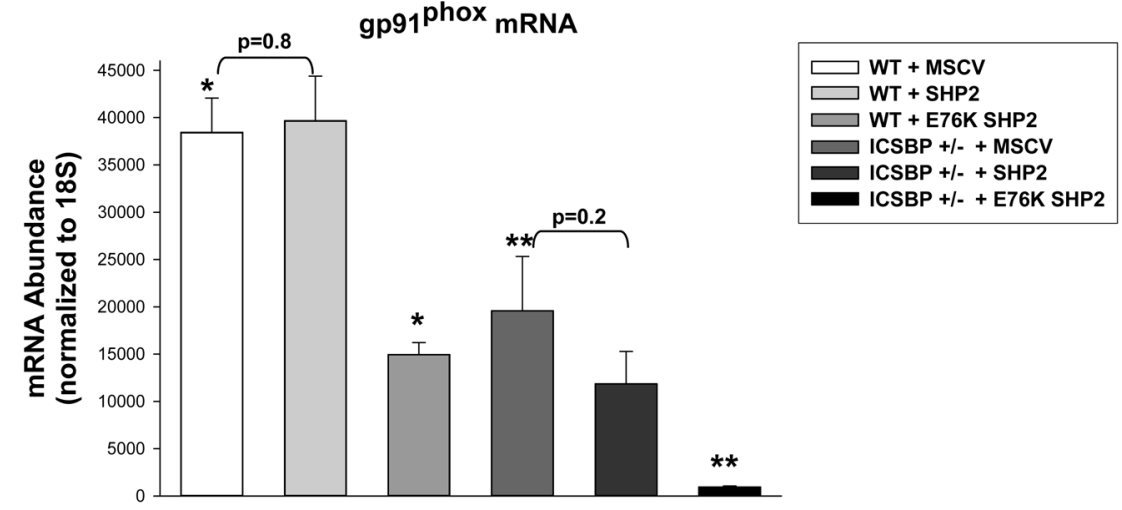

Figure 10

Constitutive SHP2 activation synergized with ICSBP deficiency to induce HSC expansion and differentiation block. Mice were analyzed after transplantation for bone marrow gene expression. (A) SHP2 was equivalently overexpressed in mice transplanted with WT or ICSBP+/- bone marrow that was transduced WT or E76K SHP2 expression vector. Expression of the SHP2 transgene was determined by real-time PCR using a primer set that detected both WT and E76K SHP2. There was no significant difference in the extent of overexpression of SHP2 or E76K SHP2 in WT $\left({ }^{*} P=0.006\right)$ or ICSBP $+/-\left({ }^{*} P=0.04\right)$ bone marrow at 24 weeks. (B) E76K SHP2 expression in ICSBP ${ }^{+/-}$bone marrow expanded the HSC and immature progenitor pool. CD34 expression in the bone marrow was determined by real-time PCR. Bone marrow CD34 expression was significantly greater in mice transplanted with control ICSBP+-- bone marrow in comparison to mice transplanted with control WT bone marrow $\left({ }^{*} P<0.02\right)$. CD34 expression was not significantly different in mice transplanted with WT bone marrow that was transduced with any of these vectors. Bone marrow CD34 expression was significantly greater in mice transplanted with E76K SHP2-expressing ICSBP $+/-$ bone marrow in comparison to ICSBP ${ }^{+/-}$controls $\left({ }^{\star \star} P=0.02\right)$. (C) E76K SHP2 expression in WT or ICSBP ${ }^{+/-}$bone marrow decreased gp91 ${ }^{\text {PHOX }}$ expression. gp91 ${ }^{\text {PHOX }}$ expression was determined by real-time PCR. Overexpression of WT SHP2 did not significantly decrease gp91 ${ }^{\text {PHOX }}$ expression in WT or ICSBP + - bone marrow. E76K SHP2 expression significantly decreased gp91 ${ }^{\text {PHOX }}$ expression in comparison to control WT $\left({ }^{\star} P=0.003\right)$ or $\mathrm{ICSBP}^{+/-}\left({ }^{\star *} P=0.008\right)$ bone marrow.

haploinsufficient bone marrow resulted in rapid AML, despite the fact that neither of these abnormalities induced AML separately.

We verified SHP2 overexpression by real-time PCR. As anticipated, there was significantly more SHP2 mRNA in the bone marrow of mice transplanted with WT or E76K SHP2 expression vectortransduced WT or ICSBP ${ }^{+/-}$bone marrow in comparison to control $(P=0.006, n=9$, and $P=0.04, n=9$, respectively) (Figure 10A). Expression of WT versus E76K SHP2 was not significantly different in $\mathrm{WT}$ or $\mathrm{ICSBP}^{+/-}$bone marrow at 24 weeks $(P=0.2, n=9$, and $P=0.7, n=9$, respectively). Expression of these 2 forms of SHP2 was not significantly different in $\mathrm{WT}$ versus $\mathrm{ICSBP}^{+/}$- bone marrow $(P=0.9, n=9)$. These results suggested that the effect of E76K SHP2 was due to the activating mutation, not overexpression.

Expression of E76K SHP2 cooperates with ICSBP deficiency to induce HSC expansion and differentiation block in vivo. To further characterize the AML that developed in these mice, we evaluated HSC and progenitor expansion by determining bone marrow CD34 expression and differentiation block by determining gp $91^{\text {PHOX }}$ expression, as above. In mice transplanted with WT bone marrow, expression of WT or E76K SHP2 did not significantly alter CD34 mRNA expres- 
A Leukemia post secondary transplant ICSBP+/-

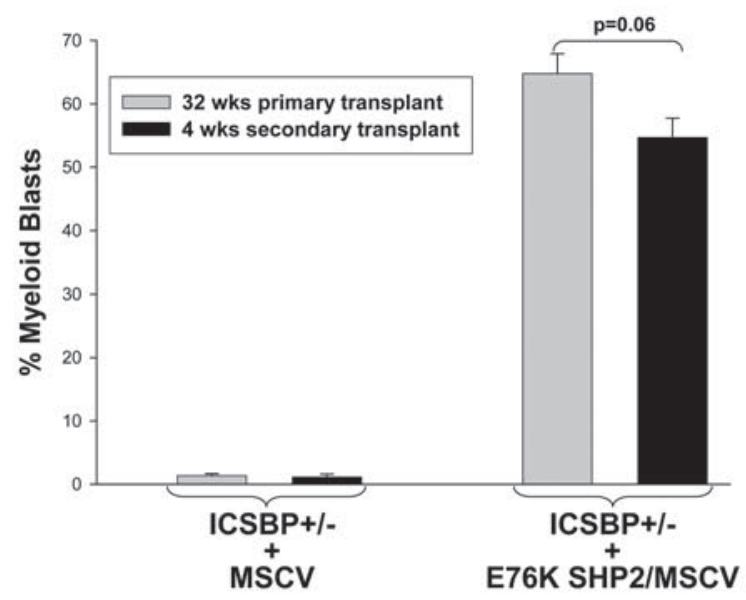

C

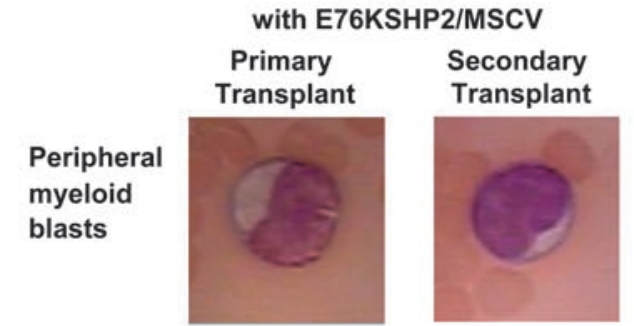

B Blood counts post secondary transplant: ICSBP+I- with MSCV or E76K SHP2

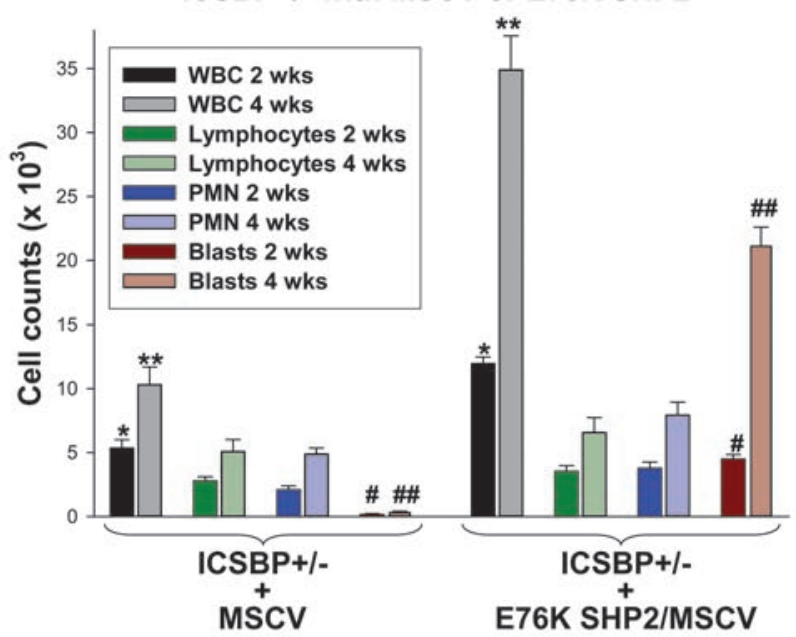

\section{Figure 11}

AML from mice transplanted with E76K SHP2-expressing ICSBP+/- bone marrow was transplantable. Irradiated WT mice were transplanted with cells isolated from mice that had been transplanted with control or E76K SHP2 expression vector-transduced ICSBP $+/$ - bone marrow. Mice were followed with peripheral blood counts every 2 weeks. (A) Secondary recipient from mice that had been transplanted with E76K SHP2-expressing $\mathrm{ICSBP}^{+/-}$bone marrow rapidly developed AML. Peripheral blood counts of mice that had been transplanted with control or E76K SHP2 expression vector-transduced ICSBP $+/-$ bone marrow were determined at 32 weeks and those of secondary recipients at 4 weeks after transplantation. The percentage of peripheral myeloid blasts was not significantly different in primary and secondary recipients with E76K SHP2-expressing ICSBP $+/-$ cells. (B) MPD and AML developed in secondary recipients of E76K SHP2-expressing ICSBP+/- hematopoietic cells, but only MPD developed in secondary recipients with control ICSBP+- cells. Peripheral blood counts were determined at 2 and 4 weeks. Mice transplanted with control ICSBP+/- cells developed a mild MPD. In contrast, E76K SHP2 expression resulted in more profound MPD and AML by 2 weeks. Significant differences in wbc are indicated by * and ${ }^{* *}$ and in blasts by \# and \#\# ( $P \leq 0.001$ for all comparisons). (C) Myeloid blasts in transplanted mice. Myeloid blasts from primary and secondary recipients of E76K SHP2-expressing ICSBP ${ }^{+/-}$hematopoietic cells are morphologically similar. Original magnification, $\times 40$.

sion $(P=0.6, n=9)$ (Figure 10B). In comparison, bone marrow CD34 expression was significantly greater in all the groups of mice transplanted with $\mathrm{ICSBP}^{+}-$bone marrow $(P<0.02, n=9)$. CD34 expression was similar in mice transplanted with control versus WT SHP2 expression vector-transduced $\mathrm{ICSBP}^{+/-}$bone marrow $(P=0.6, n=9)$. In contrast, CD34 expression was significantly greater in mice transplanted with E76K SHP2 expression vectortransduced $\mathrm{ICSBP}^{+/-}$bone marrow in comparison to the 2 control groups $(P=0.02, n=9)$. This bone marrow progenitor expansion correlated with increased circulating myeloid blasts.

Overexpression of WT SHP2 did not significantly alter gp $91^{\text {PHOX }}$ expression in mice transplanted with either WT $(P=0.8, n=9)$ or $\mathrm{ICSBP}^{+/-}$bone marrow $(P=0.2, n=9)$ (Figure 10C). In contrast, gP91 ${ }^{\text {PHOX }}$ mRNA abundance was significantly lower in mice transplanted with E76K SHP2-expressing WT or ICSBP ${ }^{+/-}$bone marrow ( $P=0.003, n=9$, and $P=0.008, n=9$, respectively). We correlated these results with respiratory burst activity in peripheral blood PMNs, as above. We found approximately half as many NBT-posi- tive, mature-appearing PMNs in mice that had received ICSBP-haploinsufficient bone marrow in comparison to WT $(50.9 \% \pm 9.2 \%$, $n=4$ ) (Figure 6D). We also found that expression of E76K SHP2 significantly decreased the percentage of NBT-positive circulating PMNs in mice transplanted with either WT or $\mathrm{ICSBP}^{+/-}$bone marrow $(P=0.006, n=4$, and $P=0.02, n=4$, respectively). The impact of E76K SHP2 expression was approximately equivalent in recipients of WT versus $\mathrm{ICSBP}^{+/-}$bone marrow.

E76K SHP2-expressing ICSBP ${ }^{+/}$leukemia was transplantable. One of the criteria for defining AML in murine models is the ability to transplant the disease into secondary recipients. In previous studies, cells from $\mathrm{ICSBP}^{-/-}$mice induced rapid leukemia in secondary recipients (15). Transplantation of $\mathrm{ICSBP}^{+/-}$cells resulted in a mild MPD, but not AML, in similar experiments. Therefore, to investigate whether E76K SHP2 expression had altered the biology of $\mathrm{ICSBP}^{+/-}$hematopoiesis and induced progression from MPD to AML, secondary transplantation experiments were performed. For these studies, mice that had been transplanted with control or 
E76K SHP2 expression vector-transduced $\mathrm{ICSBP}^{+/-}$bone marrow were sacrificed at 32 weeks. At this time, mice with E76K SHP2expressing $\mathrm{ICSBP}^{+/-}$bone marrow had at least $60 \%$ myeloid blasts in the circulation (Figure 11A), hypercellular bone marrow with myeloid blasts, and splenomegaly. Mice that had been transplanted with control $\mathrm{ICSBP}^{+/-}$bone marrow had mild MPD. Sca1-selected, B cell-depleted spleen cells were transplanted into irradiated WT mice. Peripheral blood counts were determined every 2 weeks.

By 4 weeks after transplantation, both groups had evidence of myeloproliferation (Figure 11B). Total leukocyte counts in mice transplanted with E76K SHP2-expressing $\mathrm{ICSBP}^{+/-}$cells were significantly greater than those in mice transplanted with control vector-transduced cells $(P<0.0004, n=4)$. At 4 weeks after transplantation, the percentage of circulating myeloid blasts in mice that were secondary recipients of E76K SHP2-expressing $\mathrm{ICSBP}^{+/-}$ cells was equivalent to the percentage of circulating blasts in the donors at the time of sacrifice $(P=0.06, n=4)$ (Figure 11A). These myeloid blasts were morphologically similar to blasts from the donor mice (Figure 11C). By 4 weeks, blood counts in secondary recipients of control vector-transduced $\mathrm{ICSBP}^{+/-}$cells were consistent with the mild MPD in donor mice (Figure 11B). Therefore, E76K SHP2 expression in a setting of ICSBP haploinsufficiency induced transplantable AML.

\section{Discussion}

We previously demonstrated that ICSBP tyrosine phosphorylation during myelopoiesis is required for transcription of myeloid-specific target genes and the gene encoding Nf1 $(3,7)$. We found that a constitutively active form of SHP2-PTP impairs activation of these ICSBP target genes by inhibiting ICSBP tyrosine phosphorylation in differentiating myeloid cells in vitro $(7,19)$. In the current studies, we extended these observations to examine the role of ICSBP tyrosine phosphorylation and constitutive SHP2 activation in vivo. We found that ICSBP reexpression prevented MPD and AML from developing in mice transplanted with $\mathrm{ICSBP}^{-/-}$- bone marrow. However, this reconstitution required a form of ICSBP that could be tyrosine phosphorylated. Consistent with this, we found that coexpression of a constitutively active form of SHP2 synergized with ICSBP haploinsufficiency to accelerate progression to AML in vivo. This was associated with HSC expansion and differentiation block. Therefore, these 2 leukemia-associated mutations synergized for disease progression in myeloid malignancy.

Conserved tyrosine residues in the ICSBP IRF domain are essential for activation of ets/IRF cis elements in CYBB, NCF2, and NF1 genes $(3,8)$. However, we found that these residues did not mediate the switch from ICSBP-induced repression to activation of PRDI cis elements in differentiating myeloid cells. PRDI cis elements are found in genes encoding IFN- $\beta$, the MHC class II transactivator protein, and c-myc. Therefore, non-IRF domain tyrosine residues are also likely to be relevant to regulation of myelopoiesis by ICSBP (23-25). Based on these results, we used a form of ICSBP with all tyrosines mutated to phenylalanine (Y-mut ICSBP) to investigate the impact of tyrosine phosphorylation on tumor suppression. However, introducing so many mutations may have unintended consequences on protein structure. Therefore, it was reassuring that Y-mut ICSBP bound DNA and repressed the PRDI cis element. Despite this, we were interested in finding another way to study the role of ICSBP tyrosine phosphorylation in myelopoiesis and tumor suppression. This led to our studies of constitutively active SHP2.
ICSBP is an SHP2 substrate in immature myeloid cells only but is a substrate for leukemia-associated, constitutively active SHP2 mutants throughout myelopoiesis $(3,7)$. The specific ICSBP residues that are dephosphorylated by SHP2 have not been defined, although our studies indicate that they include at least the conserved IRF domain tyrosines. In the current studies, we found that constitutive SHP2 activation cooperated with ICSBP haploinsufficiency to induce cytokine hypersensitivity and apoptosis resistance in vitro and AML in vivo. This may be related to synergistic effects of ICSBP haploinsufficiency and SHP2 activation on ICSBP target genes. Or it could represent cooperation between abnormalities in ICSBP target gene expression and effects of other SHP2 substrates. As more SHP2 substrates are identified, it will be possible to clarify the relative contributions of these various events.

Similarly, reexpression of ICSBP, but not Y-mut ICSBP, rescued MPD and delayed AML in mice transplanted with $\mathrm{ICSBP}^{-/-}$bone marrow. The impact of transduced, WT ICSBP declined over time, associated with decreased transgene expression. We hypothesize that ICSBP-/- cells with high levels of WT ICSBP expression had decreased survival, increased differentiation, or both. In contrast, Y-mut ICSBP transgene expression increased over time. This suggested that cells with high levels of Y-mut ICSBP expression might have a competitive advantage over $\mathrm{ICSBP}^{-/-}$cells. The mechanism for this will be of interest.

Our current studies focused on the transition from MPD to AML. Transformation to AML has serious consequences in human myeloid malignancies characterized by ICSBP deficiency, such as MDS and CML. This transition represents a point at which clinical intervention becomes more difficult. Identifying molecular markers of transformation would be useful for determining prognosis or identifying therapeutic targets to delay disease progression. In our studies, we quantified circulating myeloid blasts, expansion of the bone marrow $\mathrm{CD}_{3}{ }^{+}$compartment, and differentiation block as measures of progression from MPD to AML. In contrast, death was the end point for previous leukemogenesis studies using either ICSBP-deficient mice or mice expressing constitutively active SHP2. Those studies did not distinguish between death due to overwhelming MPD and death due to transformation to AML. We considered development of blast crisis (with HSC expansion and differentiation block) as more relevant and specific to the clinically important events in which we are interested. Therefore, we report development of AML instead of the more nonspecific death end point. AML that developed in our studies was characterized by progressive increase in total $\mathrm{wbc}$ and myeloid blasts and tissue infiltration with hematopoietic cells. This was similar to results that have previously been reported for the ICSBP-deficient murine leukemogenesis model.

We determined the size of the progenitor pool during disease progression by quantifying CD34 expression. Increased CD34 expression might be due to either increased numbers of CD34expressing cells or increased expression in individual cells. In preliminary studies, we verified the former explanation (data not shown). We found that CD34 expression increased during progression from MPD to AML in mice transplanted with control or Y-mut ICSBP-expressing ICSBP-/- bone marrow or E76K SHP2expressing $\mathrm{ICSBP}^{+/-}$bone marrow. CD34 expression was lower in mice transplanted with ICSBP-reconstituted ICSBP-/- bone marrow but increased with decreasing transgene expression (and disease progression), as might be expected.

Similarly, we investigated differentiation block by quantifying gp91 ${ }^{\text {PHOx }}$ expression. We found that reexpression of WT ICSBP 
rescued gp91 $1^{\text {PHOx }}$ expression in mice transplanted with ICSBP-/- $^{-/}$ bone marrow but Y-mut ICSBP expression did not. This difference decreased over time in correlation with decreased WT transgene expression and increased peripheral blasts. Similarly, decreased gp91 ${ }^{\text {PHOX }}$ mRNA in mice transplanted with E76K SHP2-expressing $\mathrm{ICSBP}^{+/-}$bone marrow correlated with $\mathrm{CD} 34$ expansion and increased myeloid blasts.

These results are of potential clinical significance, because ICSBP deficiency and constitutive SHP2 activation may coexist in human myeloid malignancies. ICSBP deficiency is found in a subgroup of tMDS/AML (12). Although the mechanism for this is unknown, studies of other IRF genes suggest that it may be related to abnormal methylation (26). Activating SHP2 mutations were found in a different study of tMDS/AML (27). ICSBP deficiency is also found in uncontrolled human CML $(14,15)$. In this disease, ICSBP deficiency may be due to abnormal cytokine expression (28). Although activating SHP2 mutations have not been described in CML, previous studies identified a role for bcr/abl in SHP2 activation (21). Therefore, bcr/abl kinase activity may induce both ICSBP deficiency and SHP2 activation. Based on current results, it will be of interest to study the coexistence of these 2 leukemia-associated abnormalities in tMDS and CML and the association with disease stage in these diseases.

\section{Methods}

Plasmids and site-directed mutagenesis. The human ICSBP cDNA was obtained from Ben-Zion Levi (Technicon Institute, Haifa, Israel) and subcloned into the pcDNAamp mammalian expression vector (with or without epitope tag) for transfection experiments and the pMSCVpuro vector for retroviral production (Stratagene), as previously described $(6,7)$. The cDNA for SHP2 PTP was obtained from Stuart Frank (University of Alabama, Birmingham, Alabama, USA) and subcloned into the pMSCVneo retroviral vector. Mutant forms of these cDNAs were generated by PCR site-directed mutagenesis using the Stratagene Quickchange protocol, as described previously (3). ICSBP with mutation of conserved IRF domain tyrosine residues to phenylalanine has been previously described (Y92/95F ICSBP) (3). A form of ICSBP with all tyrosine residues mutated to phenylalanine was also generated (referred to as Y-mut ICSBP). A leukemia-associated, activating mutation in SHP2 (E76K SHP2) was generated as described previously $(7,19)$. All PCR products were sequenced on both strands to verify that no unintended mutations had been introduced.

Oligonucleotides. Oligonucleotides were synthesized by MWG Biotech. Primers for quantitative real-time PCR were designed with the software from Integrated DNA Technologies.

Myeloid cell line culture. The human myelomonocytic cell line U937 (22) was obtained from Andrew Kraft (Hollings Cancer Center, Medical University of South Carolina, Charleston, South Carolina, USA). Cells were maintained and differentiated as described previously (2). For differentiation experiments, U937 cells were treated for 48 hours with $400 \mathrm{U} / \mathrm{ml}$ human recombinant IFN- $\gamma$ (Roche Diagnostics) (2).

Transfections and reporter gene assays. U937 cells were cultured and transfected as previously described $(1,2,7,8)$. Cells $\left(32 \times 10^{6}\right.$ per sample) were transfected with vectors to overexpress ICSBP, Y95F ICSBP, Y-mut ICSBP, or vector control; a minimal promoter/reporter vector PTATACAT with 4 copies of the -32- to -69-bp CYBB sequence (cybbTATACAT) or vector control (TATACAT); and p-CMV $\beta$ gal (to control for transfection efficiency). Transfectants were harvested 48 hours after transfection, with and without incubation with recombinant human IFN- $\gamma(400 \mathrm{U} / \mathrm{ml})$. Lysates were analyzed for CAT and $\beta$-gal activity, as described previously $(2,6)$.

Culture and retroviral transduction of primary murine bone marrow progenitors. High-titer murine stem cell retroviral supernatants were produced using the MSCVpuro and MSCVneo vectors and PT67 cell line, as per the manufacturer's instructions (Stratagene). Filtered retroviral supernatants were used immediately or stored at $-80^{\circ} \mathrm{C}$.

$\mathrm{ICSBP}^{+/-} \mathrm{C} 57 \mathrm{BL} / 6$ mice were provided by Keiko Ozato (NIH, Bethesda, Maryland, USA) (15). All murine experiments were performed with approval of the Northwestern University and Jesse Brown VA Medical Center Animal Care and Use Committees. Sca1 ${ }^{+}$bone marrow cells were obtained from the femurs of WT, ICSBP ${ }^{+-}$, or ICSBP-/- C57BL/ 6 mice using the Miltenyi magnetic bead system (Miltenyi Biotec). Cells were cultured for 24 hours in DMEM with FBS (10\%), IL-6 (10 ng/ml), IL-3 (10 ng/ml), SCF $(100 \mathrm{ng} / \mathrm{ml})$, and penicillin/streptomycin (1\%). Cells were transduced by "spinoculation," as described previously (29). Briefly, $4.0 \times 10^{6}$ cells were suspended in $3 \mathrm{ml}$ of DMEM supplemented with cytokines as above. To this was added an equal volume of retroviral supernatant and polybrene to a final concentration of $6 \mu \mathrm{g} / \mathrm{ml}$. Cells were centrifuged at $2,000 \mathrm{~g}$ for 4 hours at $15^{\circ} \mathrm{C}$, diluted 3 -fold with media, and incubated overnight. The procedure was repeated. Spinoculation reproducibly resulted in $75 \%-80 \%$ transduction under these conditions. For transplantation experiments, cells were used immediately. In other experiments, cells were cultured with puromycin $(1.2 \mathrm{ng} / \mathrm{ml})$ or G418 $(250 \mathrm{ng} / \mathrm{ml})$ in the media for 96 hours under various cytokine conditions.

Murine bone marrow transplantation. Syngenic C57BL/ 6 mice were irradiated $(5.6 \mathrm{~Gy})$, and transduced bone marrow cells $\left(0.5 \times 10^{6}\right)$ were immediately infused by retro-orbital injection. Mice were examined weekly, and any animal with greater than $10 \%$ weight loss, lethargy, or poor feeding was sacrificed. Blood counts were determined every 4 weeks on tail-bleed samples obtained using Unopette devices (BD). Differential wbc counts were performed on Wright-Giemsa-stained blood smears by counting 200 cells on duplicate slides. Cohorts of mice ( 6 total) were sacrificed at 16 and 24 weeks. Bone marrow from each animal was expanded for 24 hours in DMEM with FBS (10\%), GM-CSF (10 ng/ml), IL-3 (10 ng/ml), and penicillin/streptomycin (1\%). Viable cells were recovered by a magnetic bead separation technique (Miltenyi Biotec).

In other studies, 3 mice transplanted with $\mathrm{ICSBP}^{+/-}$bone marrow that had been transduced with control or E76K SHP2 expression vector were sacrificed at 32 weeks. Sca1 ${ }^{+}$, B cell-depleted spleen cells were isolated and cultured in IL-3, IL- 6 , and SCF for 48 hours, as described above. These cells $\left(1 \times 10^{6}\right)$ were transplanted into irradiated WT mice, and peripheral blood leukocyte counts were determined after transplantation, as described above.

Ex vivo differentiation of murine bone marrow cells. Bone marrow cells were obtained from WT, $\mathrm{ICSBP}^{+/-}$, or $\mathrm{ICSBP}^{-/-} \mathrm{C} 57 \mathrm{BL} / 6$ mice and cultured, as described above. After 48 hours, cells were either harvested or transferred to DMEM supplemented with FBS (10\%), GM-CSF (10 ng/ml), IL-3 $(10 \mathrm{ng} / \mathrm{ml})$, and penicillin/streptomycin (1\%). Cells were cultured at $37^{\circ} \mathrm{C}$ and $5 \% \mathrm{CO}_{2}$ for 48 hours. Some cells were transferred to DMEM supplemented with FBS (10\%), G-CSF (10 ng/ml), and penicillin/streptomycin (1\%). Cells were harvested after 72 hours. Cells were analyzed for Sca1 expression by flow cytometry and real-time PCR.

Proliferation assays. Proliferation assays were performed as previously described $(6,7)$. Briefly, cells were harvested and plated at $10^{5}$ cells per $200 \mu \mathrm{l}$ in a 96-well dish in DMEM supplemented with antibiotics and IL-3, but without GM-CSF. After 48 hours of deprivation, media was supplemented with a serial dilution of GM-CSF (10-fold from $10 \mathrm{ng} / \mathrm{ml}$ to $0.01 \mathrm{ng} / \mathrm{ml}$ vs. no cytokine). Cells were stimulated with GM-CSF for 24 hours at $37^{\circ} \mathrm{C}, 5 \%$ $\mathrm{CO}_{2} \cdot\left[{ }^{3} \mathrm{H}\right]$ thymidine was added for the last 8 hours of culture. Cells were harvested with an automated cell harvester and ${ }^{3} \mathrm{H}$ incorporated into DNA counted by scintillation counting, according to standard techniques.

Apoptosis assays. Annexin V/propidium iodide (annexin V/PI) (Beckman Coulter) double staining was used according to the manufacturer's instruc- 
tions. The cells were adjusted to a concentration of $1 \times 10^{6} \mathrm{cells} / \mathrm{ml}$ and incubated with annexin V-FITC solution $(2.5 \mu \mathrm{g} / \mathrm{ml})$ and PI $(12.5 \mu \mathrm{g} / \mathrm{ml})$ on ice for 15 minutes and analyzed on a BD FACScan flow cytometer.

Western blots. U937 cells or murine bone marrow cells were lysed by boiling in $2 \times$ SDS sample buffer. Lysate proteins $(50 \mu \mathrm{g})$ were separated by SDSPAGE and transferred to nitrocellulose according to standard techniques. Western blots were serially probed with antibodies against ICSBP, SHP2, and GAPDH (to control for loading), as described previously $(2,3,6,7)$.

In other studies, total cell lysates, nuclear proteins, or IVT proteins were immunoprecipitated under denaturing conditions with anti-phospho-tyrosine antibody, anti-epitope tag antibody, or control antibody. Immunoprecipitates were collected with Staphylococcus aureus protein A agarose or protein $\mathrm{G}$ sepharose and separated by SDS-PAGE. In studies with cellular proteins, Western blots were probed with ICSBP antibody, similar to previously described studies $(3,7)$. IVT proteins were identified by autoradiography of SDS-PAGE, as previously described $(3,6)$.

EMSA. IVT WT and Y-mut ICSBP were generated in rabbit reticulocyte lysate, as previously described $(3,7)$. For some studies, proteins were labeled by inclusion of $\left[{ }^{35} \mathrm{~S}\right]$ methionine in the synthesis reaction. Binding assays were performed with a radiolabeled probe representing the PRDI consensus sequence (similar to refs. 3, 7). Some IVT proteins were preincubated with recombinant Yop-PTP, as previously described (3). Some binding assays were preincubated with ICSBP antibody or preimmune serum (as described in refs. 3,6 ).

1. Nelson, N., Marks, M.S., Driggers, P.H., and Ozato, K. 1993. Interferon consensus sequence-binding protein, a member of the interferon regulatory factor family, suppresses interferon-induced gene transcription. Mol. Cell. Biol. 13:588-599.

2. Eklund, E.A., Jalava, A., and Kakar, R. 1998. PU.1, interferon regulatory factor 1 , and interferon consensus sequence-binding protein cooperate to increase gp91(phox) expression. J. Biol. Chem. 273:13957-13965.

3. Kautz, B., Kakar, R., David, E., and Eklund, E.A. 2001. SHP1 protein-tyrosine phosphatase inhibits gp91PHOX and p67PHOX expression by inhibiting interaction of PU.1, IRF1, interferon consensus sequence-binding protein, and CREB-binding protein with homologous cis elements in the $C Y B B$ and NCF2 genes. J. Biol. Chem. 276:37868-37878.

4. Rehli, M., et al. 2000. PU.1 and interferon consensus sequence-binding protein regulate the myeloid expression of the human Toll-like receptor 4 gene. J. Biol. Chem. 275:9773-9781.

5. Wang, I.M., et al. 2000. An IFN-gamma-inducible transcription factor, IFN consensus sequence binding protein (ICSBP), stimulates IL-12 p40 expression in macrophages. J. Immunol. 165:271-279.

6.Zhu, C., Saberwal, G., Ly, Y.F., Platanias, L.C., and Eklund, E.A. 2004. The interferon consensus sequence-binding protein activates transcription of the gene encoding Neurofibromin 1. J. Biol. Chem. 279:50874-50885.

7. Huang, W., et al. 2006. Leukemia-associated, constitutively active mutants of SHP2 protein tyrosine phosphatase inhibit NF1 transcriptional activation by the interferon consensus sequence binding protein. Mol. Cell. Biol. 26:6311-6332.

8. Huang, W., Horvath, E., and Eklund, E.A. 2007. PU.1, interferon regulatory factor 2 , and the interferon consensus sequence binding protein cooperate to activate NF1 transcription in differentiating myeloid cells. J. Biol. Chem. 282:6629-6643.

Real-time PCR. RNA was isolated using the TRIzOL reagent, according to the manufacturer's instructions (Invitrogen), and tested for integrity by denaturing gel electrophoresis. Real-time PCR was performed using SYBR green according to the "standard curve" method, as per manufacturer's instructions. Results were normalized to 18S. Experiments were performed in triplicate. For transplanted mice, bone marrow from each animal was analyzed individually.

Statistics. Significance of differences between 2 groups was determined by 2 -tailed Student's $t$ test and between more than 2 groups by ANOVA. $P$ values of less than or equal to 0.05 were considered significant.

\section{Acknowledgments}

This work was supported by a Veteran's Health Administration Merit review (to E.A. Eklund), by NIH R01-CA095266 (to E.A. Eklund), and by a Translational Research Award from the Leukemia and Lymphoma Society of America.

Received for publication August 27, 2007, and accepted in revised form November 28, 2007.

Address correspondence to: Elizabeth A. Eklund, Northwestern University Feinberg School of Medicine, 710 N. Fairbanks Court, Olson Pavilion, Room 8524, Chicago, Illinois 60611, USA. Phone: (312) 5034625; Fax: (312) 908-5717; E-mail: e-eklund@northwestern.edu.

9. Scheller, M., et al. 1999. Altered development and cytokine responses of myeloid progenitors in the absence of transcription factor, interferon consensus sequence binding protein. Blood. 94:3764-3771.

10. Tamura, T., Thotakura, P., Tanaka, T.S., Ko, M.S.H., and Ozato, K. 2005. Identification of target genes and a unique cis element regulated by IRF8 in developing macrophages. Blood. 106:1938-1947.

11. Sharf, R., et al. 1997. Phosphorylation events modulate the ability of ICSBP to interact with IRFs and to bind DNA. J. Biol. Chem. 272:9785-9792.

12. Qian, Z., Fernald, A.A., Godley, L.A., Larson, R.A., and Le Beau, M.M. 2002. Expression profiling of CD34+ hematopoietic stem/progenitor cells reveals distinct subtypes of therapy related acute myeloid leukemia. Proc. Natl. Acad. Sci. U. S. A. 99:14925-14930.

13. Schmidt, M., et al. 1998. Lack of interferon consensus sequence binding protein (ICSBP) transcripts in human myeloid leukemias. Blood. 91:22-29.

14. Schmidt, M., Hochhaus, A., Nitsche, A., Hehlmann, R., and Neubauer, A. 2001. Expression of nuclear transcription factor interferon consensus sequence binding protein in chronic myeloid leukemia correlates with pretreatment risk features and cytogenetic response to interferon-alpha. Blood. 97:3648-3650.

15. Holtchke, T., et al. 1996. Immunodeficiency and chronic myelogenous leukemia-like syndrome in mice with a targeted mutation of the ICSBP gene. Cell. 87:307-317.

16. Bentires-Ali, M., et al. 2004. Activating mutations of the Noonan syndrome-associated SHP2/PTPN11 gene in human solid tumors and adult acute myelogenous leukemia. Cancer Res. 64:8816-8820.

17. Tartaglia, M., et al. 2003. Somatic mutations in PTPN11 in juvenile myelomonocyticleukemia, myelodysplastic syndromes and acute myeloid leukemia. Nat. Genet. 34:148-150.

18. Keilhack, H., David, F.S., McGregor, M., Cantley, L.C., and Neel, B.G. 2005. Diverse biochemical properties of Shp2 mutants. Implications for dis- ease phenotypes. J. Biol. Chem. 280:30984-30993.

19. Lindsey, S., et al. 2007. Activation of SHP2 proteintyrosine phosphatase increases HoxA10-induced repression of the genes encoding gp91(PHOX) and p67(PHOX). J. Biol. Chem. 282:2237-2249.

20. Sattler, M., et al. 2002. Critical role for Gab2 in transformation by Bcr/abl. Cancer Cell. 1:479-492.

21. Scherr, M., et al. 2006. Enhanced sensitivity to inhibition of SHP2, Stat5 and Gab2 expression in chronic myeloid leukemia. Blood. 107:3279-3287.

22. Larrick, J.W., Anderson, S.J., and Koren, H.S. 1980. Characterization of a human macrophage-like cell line stimulated in vitro: a model of macrophage functions. J. Immunol. 125:6-14.

23. Keller, B., Chee, K.I., Kim, T.H., and Maniatis, T. 1991. Identification and characterization of a novel repressor of Beta interferon gene expression. Genes Dev. 5:868-879.

24. Kakkis, E., Riggs, K.I., Gillespie, W., and Calame, K. 1989. A transcriptional repressor of c-myc. Nature. 339:718-721.

25. Chang, D.H., Angelin-Cuclos, C., and Calame, K. 2000. BLIMP-1: trigger for differentiation of myeloid lineage. Nat. Immunol. 1:169-176.

26. Ortmann, C.A., et al. 2005. Down regulation of interferon regulatory factor 4 gene expression in leukemic cells due to hypermethylation of CpG motifs in the promoter region. Nucleic Acids Res. 33:6895-6905.

27. Christiansen, D.H., Desta, F., Andersen, M.K., and Pedersen-Bjergaard, J. 2007. Mutations of the PTPN11 gene in therapy related MDS and AML with rare balanced chromosome translocations. Genes Chromosomes Cancer. 46:517-521.

28. Aswald, J.M., Lipton., J.H., and Messmer, H.A. 2002. Intracellular cytokine analysis of interferon gamma in T cells of patients with chronic myeloid leukemia. Cytokines Cell. Mol. Ther. 7:75-82.

29. Bahnson, A.B., et al. 1995. Centrifugal enhancement of retroviral mediated gene transfer. J. Virol. Methods. 54:131-143. 